\title{
Renormalization group in super-renormalizable quantum gravity
}

\author{
Leonardo Modesto $^{1, \mathrm{a}}$, Lesław Rachwal ${ }^{1,2, \mathrm{~b}}{ }_{(\mathbb{D})}$, Ilya L. Shapiro ${ }^{3,4,5, \mathrm{c}}$ \\ ${ }^{1}$ Department of Physics, Southern University of Science and Technology, Shenzhen 518055, China \\ 2 Instituto de Física, Universidade de Brasília, Brasília, DF 70910-900, Brazil \\ ${ }^{3}$ Departamento de Física, ICE, Universidade Federal de Juiz de Fora, Campus Universitário, Juiz de Fora, MG 36036-330, Brazil \\ ${ }^{4}$ Tomsk State Pedagogical University, Tomsk 634041, Russia \\ 5 Tomsk State University, Tomsk 634050, Russia
}

Received: 11 May 2018 / Accepted: 28 June 2018 / Published online: 6 July 2018

(C) The Author(s) 2018

\begin{abstract}
One of the main advantages of super-renormalizable higher derivative quantum gravity models is the possibility to derive exact beta functions, by making perturbative one-loop calculations. We perform such a calculation for the Newton constant by using the Barvinsky-Vilkovisky trace technology. The result is well-defined in a large class of models of gravity in the sense that the renormalization group beta functions do not depend on the gauge-fixing condition. Finally, we discuss the possibility to apply the results to a large class of nonlocal gravitational theories which are free of massive ghost-like states at the tree-level.
\end{abstract}

\section{Introduction}

The calculation of quantum corrections always had a very special role in quantum theories of gravity. The first relevant calculation was done by t'Hooft and Veltman [1], who derived the one-loop divergences in the quantum version of general relativity, including coupling to the minimal scalar field. Soon after similar calculations were performed for gravity-vector and gravity-fermion systems [2,3]. These first calculations have a great merit, regardless of the fact that the output was shown to be gauge-fixing dependent [4]. Later on one could learn a lot from the two-loop calculations in general relativity $[5,6]$. Technically more complicated are calculations in four-derivative gravity, which were first performed in [7] and with some corrections in [8-11] and finally in [12], where some extra control of the calculations was introduced and the hypothesis of the non-zero effect of the topological Gauss-Bonnet term [13] explored. Let us also

\footnotetext{
a e-mail: 1modesto@ sustc.edu.cn

b e-mail: grzerach@gmail.com

c e-mail: shapiro@fisica.ufjf.br
}

mention similar calculations in the conformal version of the four-derivative theory $[8,14,15]$.

The importance of four-derivative quantum gravity is due to its renormalizability [16], which is related to the presence of massive unphysical ghosts, typical in the higher derivative field theories. Naturally, there were numerous and interesting works trying to solve the unitarity problem in this theory [17-21]. The mainstream approach is based on the expectation that the loop corrections may transform the real massive unphysical pole in the tensor sector of the theory into a pair of complex conjugate poles, which do not spoil unitarity within the Lee-Wick quantization scheme [22,23]. However, it was shown that the definite knowledge of whether this scheme works or not requires an exact non-perturbative beta function for the coefficient of the Weyl-squared term and for the Newton constant [24]. The existing methods to obtain such a non-perturbative result give some hope [25,26], but unfortunately they are not completely reliable ${ }^{1}$ and, therefore, the situation with unitarity in the four-derivative quantum gravity is not certain, at least.

Another interesting aspect of quantum corrections in models of gravity is related to the running of the cosmological constant $\Lambda_{\mathrm{cc}}$ and especially Newton constant $G$. These quantum effects may be relevant in cosmology and astrophysics (see, e.g. $[28,29]$ ) and can be explored in different theoretical frameworks, such as semiclassical gravity [30,31], higher derivative quantum gravity $[8,20]$, low-energy effective quantum gravity [32-34], induced gravity $[35,36]$ and functional renormalization group [37]. Indeed, the status of the corresponding types of quantum corrections is different, but there are also some common points. In particular, in many cases one can formulate general restrictions on the running

\footnotetext{
${ }^{1}$ One of the reasons is a strong gauge-fixing dependence of the on-shell average effective action, which was discussed in Yang-Mills theory [27] and is expected to take place also in quantum gravity.
} 
of the Newton constant $G$, which are based on covariance and dimensional arguments [38]. The beta function for the inverse Newton constant which follows from these condition has the form

$\mu \frac{d}{d \mu} \frac{1}{G}=\sum_{i j} A_{i j} m_{i} m_{j}$,

where $m_{i}$ are masses of the fields or more general parameters in the action with the dimensions of masses and $A_{i j}$ are given by series in coupling constants of the theory.

In the perturbative quantum gravity case there may be one more complication. In the model based on EinsteinHilbert's gravity there is no beta function for $G$, and in the four-derivative model this beta function is dependent on the choice of gauge-fixing condition [8,39-42]. Only a dimensionless combination of $G$ and $\Lambda_{\mathrm{cc}}$ has well-defined running, but this is not sufficient for the mentioned applications to cosmology and astrophysics.

Recently there was a significant progress in development of perturbative quantum gravity models which have very different properties. If the action of the theory includes local covariant terms that have six or more derivatives, this theory may possess a few remarkable properties, as listed below. First of all, such a theory is super-renormalizable [43] (see also earlier works $[44,45])$. Secondly, there are formal arguments showing that the theory can be formulated as unitary, in the case that there are only complex conjugate massive poles in the tree-level propagator $[46,47]$. Moreover, the theory is unitary at any order in the perturbative loop expansion when the CLOP prescription [48,49] is implemented or non-equivalently when the theory is defined through a non-analytic Wick rotation from Euclidean to Minkowskian signature. The third important advantage is that the theories with more than six derivatives may have gauge-fixing and field reparametrization independent beta functions for both $G$ and $\Lambda_{\mathrm{cc}}$. There are also serious indications that the problem of stability of the theory with complex poles can be resolved within the Lee-Wick approach. In order to avoid lengthy discussion of this issue (which has no direct relation to the main subject of the paper) in the Introduction, we devote to it an Appendix C.

All this means that such a theory satisfies the minimal set of consistency conditions and deserves a detailed investigation at both classical and quantum levels. The classical aspects of the theory started to be explored recently in [50], where it was shown that the version with real simple poles has no singularity in the modified Newtonian potential. Quite recently this result was generalized for more general cases including multiple and complex poles [51,52]. Furthermore, in [51,53] the detailed analysis of light bending in six-derivative models was given. Another generalization of simple higher derivative model is nonlocal gravity, where we allow for nonlocal functions of differential operators [54-56].
Moreover, there exists a class of nonlocal theories in which UV behaviour is exactly the same like in polynomial higher derivative theories. Therefore, they also satisfy the above three points, namely they are quantum super-renormalizable unitary models and the analysis of divergences and RG running presented here apply to these theories as well.

Until now, the unique example of quantum calculations in the super-renormalizable quantum gravity was the derivation of the beta function for the cosmological constant in [43]. Here we start to explore the models further and derive the most relevant phenomenologically one-loop beta function for the Newton constant $G$.

The work is organized as follows. In Sect. 2 one can find a brief general review of the super-renormalizable models [43], including power counting and gauge-fixing independence of the beta functions. In Sect. 3, we describe the one-loop calculations. Some of the relevant bulky formulas are separated into Appendix A, to provide a smooth reading of the main text. In Sect. 4, two important classes of the nonlocal models of quantum gravity are considered. It turns out that the derivation of one-loop divergences by taking a limit in the results for a polynomial models meets serious difficulties, which can be solved only for a special class of nonlocal theories, which are asymptotically polynomial in the ultraviolet regime. But still these theories are super-renormalizable or even finite. In Sect. 5, the renormalization group for the Newton and cosmological constants are discussed, within the minimal subtraction scheme of renormalization. Finally, in Sect. 6 we draw our conclusions and outline general possibilities for further work.

\section{Six and higher derivative quantum gravity}

One of the simplest models of super-renormalizable quantum gravity is based on the action

$$
\begin{aligned}
S_{N}= & \int d^{4} x \sqrt{|g|}\left\{\omega_{N, R} R \square^{N} R+\omega_{N, C} C \square^{N} C\right. \\
& +\omega_{N, \mathrm{~GB}} \mathrm{~GB}_{N}+\omega_{N-1, R} R \square^{N-1} R \\
& +\omega_{N-1, C} C \square^{N-1} C+\omega_{N-1, \mathrm{~GB}} \mathrm{~GB}_{N-1}+\cdots \\
& \left.+\omega_{0, R} R^{2}+\omega_{0, C} C^{2}+\omega_{0, \mathrm{~GB}} \mathrm{~GB}_{0}+\omega_{\mathrm{EH}} R+\omega_{\mathrm{cc}}\right\} .
\end{aligned}
$$

Here $R$ is the scalar curvature and $C$ is the Weyl tensor (with writing of all indices suppressed), e.g.

$C \square^{n} C=$ Riem $\square^{n}$ Riem -2 Ric $\square^{n}$ Ric $+\frac{1}{3} R \square^{n} R$.

In the last formula Riem and Ric stand for the Riemann and Ricci tensors (with writing of all indices sup- 
pressed again), correspondingly. Furthermore, the generalized Gauss-Bonnet (GB) term is

$\mathrm{GB}_{n}=$ Riem $\square{ }^{n}$ Riem -4 Ric $\square^{n}$ Ric $+R \square^{n} R$,

which is not topological for $n \neq 0$. It can be easily shown that this term can be reduced to the $\mathcal{O}\left(\mathrm{Riem}^{3}\right)$ terms for these values of $n$. We also notice that for $n=0$ and in $d=4$ dimensions any order symmetrized variation of the GB term is a total derivative $[1,13]$ hence it does not contribute at all. The coefficient of the Einstein-Hilbert term and the density of the cosmological constant term are denoted as $\omega_{\mathrm{EH}}=-1 /(16 \pi G)$ and $\omega_{\mathrm{cc}}=-\Lambda_{c c} /(8 \pi G)$, in order to provide homogeneous notation. Action (2) is the most general one which is at most quadratic in curvatures. One can extend it preserving power counting by adding extra terms that are of the higher orders in the curvature tensor [57]. As we shall discuss in what follows, such $\mathcal{O}\left(\mathrm{Riem}^{3}\right)$-terms are not necessary for super-renormalizability and in this sense they represent the non-minimal sector of the theory. In what follows we will see that the Einstein-Hilbert and cosmological terms are relevant, but for $N \geqslant 2$ they do not affect the divergences.

It proves useful to discuss the dimensions of the parameters included into the action (2). Standard considerations show that the dimensions of the parameters are

$$
\begin{aligned}
{\left[\omega_{N, R}, \omega_{N, C}, \omega_{N, \mathrm{~GB}}\right] } & ={\text { (mass })^{-2 N},} \\
{\left[\omega_{N-1, R}, \omega_{N-1, C}, \omega_{N-1, \mathrm{~GB}}\right] } & ={\text { (mass })^{-2 N+2} .}^{-2 N} .
\end{aligned}
$$

Therefore, by dimensional reasons similar to the ones that lead to (1), one can show that the beta function for the inverse Newton constant should be proportional to the ratios $\omega_{N-1, i} / \omega_{N, j}$, where $i, j=(R, C, \mathrm{~GB})$ for $N \geqslant 2$. In what follows this conclusion will be supported by power counting and by the direct calculation of the one-loop counterterms.

Let us consider the power counting in the theory (2) which is equivalent to the most general local theory with $2 N+$ 4 derivatives of the metric. The formula for the superficial degree of divergence $D$ for a $p$-loop diagram follows from a general expression

$D+d=\sum_{l=1}^{l_{\text {int }}}\left(4-r_{l}\right)-4 n_{v}+4+\sum_{v} K_{v}$

and the topological relation

$l_{\text {int }}=p+n_{v}-1$.

Here $d$ is the number of derivatives on the external lines of the diagrams, $r_{l}$ is the power of momenta in the inverse of the propagators, $K_{v}$ is the power of momenta in the given vertex $v, n_{v}=\sum_{v}$ is the total number of vertices and $l_{\text {int }}$ is the number of internal lines of the diagram. One can introduce the gauge-fixing condition in the theory (2) in such a way that effectively $r_{l} \equiv 2 N+4$ (for this one can check [43] and the next section of the present work for the details). The strongest divergences come from the largest $K_{v}$ and hence one can set $K_{v}=r_{l}=2 N+4$, without losing generality. Then it is easy to arrive at the result

$D+d=4+2 N(1-p)$.

For the logarithmic divergences $(D=0)$ we get the estimate for the dimension of the $p$-loop counterterms, $d=$ $4+2 N(1-p)$. The last expressions have several important consequences.

First of all, from (8) one learns that the theories with $N \geqslant$ 1 are super-renormalizable, since only diagrams with $p=$ 1,2, 3 may be divergent. Moreover, for $N \geqslant 3$ only oneloop diagrams may be divergent. As a result, the one-loop beta functions will be exact in the theories with $N \geqslant 3$.

Second, since the divergences come from $p \geqslant 1$, it is clear that for any $N \geqslant 1$ the dimension of the counterterms can only be $d=4,2,0$. As a result, the terms which are included in the first two lines of the action (2) are not subjected to the renormalization procedure for $N \geqslant 2$. The multiplicative renormalizability requires that the action (2) includes the terms in the last line, which we can also parametrize as

$S_{\text {add }}=S_{\mathrm{EH}}+S_{4 D}$,

where

$S_{\mathrm{EH}}=-\frac{1}{16 \pi G} \int d^{4} x \sqrt{|g|}\left\{R+2 \Lambda_{\mathrm{cc}}\right\}$

is the Einstein-Hilbert action with the cosmological constant and

$S_{4 D}=\int d^{4} x \sqrt{|g|}\left\{a_{1} C^{2}+a_{2} E+a_{3} \square R+a_{4} R^{2}\right\}$

is the action with four-derivative terms. We included the surface term $\square R$, along with the Euler density term $E$.

Third, for $N \geqslant 2$ the renormalizations of the parameters $a_{1,2,3,4}$ and $G, \Lambda_{\mathrm{cc}}$ of the actions (10) and (11) do not depend on these parameters of the action, but only on the parameters of the higher derivative terms of the action (2) (first two lines of it). Let us note that the renormalization of $\Lambda_{\mathrm{cc}} /(8 \pi G)$ was described in details in [43] and therefore we will not discuss its details here. As far as we intend to calculate the beta function for the Newton constant $G$, it is sufficient to consider only the quantum effects of the theory with the action (2). Indeed, the result can be modified by adding $\mathcal{O}\left(\mathrm{Riem}^{3}\right)$-type terms [43,47,57-59] (sometimes called "killers" [57] for the ability to make the theory free of any divergence), but we will not consider these terms here. One can note that the remaining terms, which do not affect the divergences, can provide the complex massive poles and hence lead to the unitarity in the Lee-Wick sense. Therefore, the theory (2) turns out to be quite general and deserves an explicit calculation of the physically relevant beta function for $G$. 
Fourth, an important observation is that for $N \geqslant 1$ the classical equations of motion in the theory have $4+2 N \geqslant 6$ derivatives, while the divergences are removed by the counterterms which have 0,2 and 4 derivatives. It is well-known that the gauge-fixing dependence of the one-loop counterterms is always proportional to the classical equations of motion. The general proof of this statement can be found in [60], and one can find a specific proof for the one-loop contributions in the book [61]. The general theorem enables one to establish gauge dependence in second- and fourth-order quantum gravity without explicit calculations [8,39]. And in the case of six- or higher than six-derivative models the same statements guarantee the gauge-fixing independence of all divergences.

Finally, we conclude that it is completely consistent to calculate only the beta function for $G$, without deriving the beta functions for the coefficients of the four-derivative terms $a_{1,2,3,4}$. Moreover, the calculation of the beta function for $G$ can be performed in any useful gauge, because the result does not depend on the choice of gauge-fixing.

\section{One-loop calculation}

The calculation of one-loop divergences can be performed within the background field method. The splitting into background and quantum metric is as follows:

$g_{\mu \nu} \rightarrow g_{\mu \nu}^{\prime}=g_{\mu \nu}+h_{\mu \nu}$

The Lagrangian quantization is based on the Faddev-Popov scheme requires introducing the gauge-fixing condition $\chi_{\mu}$ and the weight operator $C^{\mu \nu}$.

The one-loop effective action is given by expression [8]

$$
\begin{aligned}
\bar{\Gamma}^{(1)}\left(g_{\mu \nu}\right)= & \frac{i}{2} \ln \operatorname{Det} \hat{\mathcal{H}}-\frac{i}{2} \ln \operatorname{Det} \hat{\tilde{C}} \\
& -i \ln \operatorname{Det} \hat{\mathcal{H}}_{\mathrm{gh}},
\end{aligned}
$$

where $\hat{\mathcal{H}}$ is the bilinear form of the action (2) with the gaugefixing term added with respect to gravitational perturbations $h_{\mu \nu}$, and $\hat{\mathcal{H}}_{\mathrm{gh}}$ is the bilinear form of the action of the gauge ghosts. $\hat{\tilde{C}}$ is the bilinear form of the gauge-fixing action with respect to gauge-fixing conditions, which is related to the weight operator. All operators in (13) are second variational derivatives of the corresponding actions and therefore are Hermitian. Moreover, they should be divided by the corresponding powers of the renormalization parameter $\mu$ in the dimensional regularization, which are not shown in the last formula. Also, in order to have homogeneous propagators for the quantum metric and the ghosts, one can, e.g. replace $\hat{\mathcal{H}}_{\text {gh }}=M_{\alpha}{ }^{\beta}$ by the product $\tilde{C}^{\gamma \alpha} M_{\alpha}{ }^{\beta}$, while the coefficient of the weight operator contribution in (13) changes from $-i / 2$ to $i / 2$.
In this paper the one-loop computations have been done in Euclidean signature. Indeed, for the Lee-Wick gravitational theories, which we proposed for the first time in [46,47], we can have extra nonlocal divergences in Minkowski space due to the Wick rotation in the theory with complex poles. It is important to note that these divergences have nothing to do with the UV limit and renormalization group which we deal with here. At the same time, no problems of this kind exist for the theories with real poles.

Let us expand on the case of complex conjugate poles. For Lee-Wick theories the continuation to Minkowski space is guaranteed consistently with perturbative unitarity by the procedure described below.

The CLOP prescription [48] (due to Cutkosky, Landshoff, Olive, and Polkinghorne) gives a Lorentz-invariant and unitary result. It consists on taking the masses of the complex conjugate poles to be unrelated complex mass parameters to avoid the overlap of the poles for finite values of the external energy. At the end of the computation one has to impose the condition that the poles are complex conjugate to each other. In particular, the CLOP prescription allows to make the Wick rotation to Euclidean signature, as explained in [49]. The CLOP prescription does not affect the renormalization of the theory because the divergent contributions to the quantum effective action have a regular limit when we impose the square masses to be complex conjugate each other. The CLOP prescription gives an unambiguous result in simple diagrams, as confirmed by the explicit calculation of the bubble diagram in [49]. However, in [48] it is shown that there are ambiguities at higher order in the loop expansion.

Let us note that the described complications concern only the case when the massive poles are complex conjugate, while for the local theories with real ghosts there is no need to worry about the Lee-Wick quantization or about continuation to Minkowski space, since unitarity is already violated at treelevel.

\subsection{Gauge-fixing and relevant operators}

The general form of the gauge-fixing action is

$S_{\mathrm{gf}}=\int d^{4} x \sqrt{|g|} \chi_{\mu} C^{\mu v} \chi_{\nu}$,

where the gauge condition is

$\chi_{\mu}=\nabla^{\lambda} h_{\lambda \mu}-\beta \nabla_{\mu} h$

Here and below the covariant derivatives are constructed with the background metric. It is sufficient for us to take the weight function $C^{\mu \nu}$ in the following form

$C^{\mu \nu}=-\frac{1}{\alpha}\left(g^{\mu v} \square+\gamma \nabla^{\mu} \nabla^{v}-\nabla^{v} \nabla^{\mu}\right) \square^{N}$. 
By $\tilde{C}^{\mu \nu}$ in (13) we mean the self-adjoint extension of the operator $C^{\mu \nu}$. The action of the gauge ghosts $\bar{c}^{\mu}$ and $c_{\nu}$ has a standard form

$S_{\mathrm{gh}}=\int d^{4} x \sqrt{|g|} \bar{c}^{\mu} M_{\mu}{ }^{\nu} c_{\nu}$,

the operator $M$ is defined as usual (see, e.g., [61], because in this part there is no essential difference with the fourderivative quantum gravity), such that we arrive at

$M_{\mu}^{\nu}=\delta_{\mu}^{v} \square+\nabla^{\nu} \nabla_{\mu}-2 \beta \nabla_{\mu} \nabla^{\nu}$.

At that point one can observe the first essential simplification coming from our interest in the Einstein-Hilbert counterterm only. For the gauge-fixing action (14) with (15) and (16), both the weight operator $\tilde{C}^{\mu \nu}$ and the bilinear operator of the ghost action $M_{\mu}{ }^{\nu}$ in (18) are homogeneous functions of covariant derivative $\nabla_{\alpha}$ and do not include dimensional parameters. As a result, the corresponding functional determinants contribute only to the divergences of the four-derivative terms in (11). Since we are interested in the beta function for the Newton constant only, we can concentrate our attention only on the first term in the expression (13) and do not pay attention on the other two terms.

Let us consider the relevant first term in (13). We remember, once again, that the final result for divergences does not depend on the choice of $\chi_{\mu}$ and $C^{\mu \nu}$. Therefore, these functions can be defined to make calculations as simple as possible. The primary role of the gauge-fixing is to make the operator $\hat{\mathcal{H}}$ non-degenerate and this can be achieved for different values of the gauge-fixing parameters $\alpha, \beta$, and $\gamma$ in Eqs. (15) and (16). Due to the gauge-fixing independence of the one-loop effective action one can choose the gauge-fixing parameters in such a way that operator $\hat{\mathcal{H}}$ assumes the minimal form. This means that the highest-order derivatives form the combination $\square^{N+2}$. The corresponding calculations are essentially the same as in the four-derivative quantum gravity.

The requirement of minimality leads to the following values:

$\alpha=\frac{2}{\omega_{N, C}}, \quad \beta=\frac{\omega_{N, C}-6 \omega_{N, R}}{4 \omega_{N, C}-6 \omega_{N, R}}$,

$\gamma=\frac{2 \omega_{N, C}-3 \omega_{N, R}}{3 \omega_{N, C}}$.

One can see that the gauge-fixing parameters $\beta$ and $\gamma$ depend only on the ratio $\omega_{N, R} / \omega_{N, C}$ and are independent on other coefficients appearing in the gravitational action (2). In particular, the minimal choice of the gauge-fixing conditions does not depend on the generalized Gauss-Bonnet term, namely $\omega_{N, \mathrm{~GB}}$. This is obviously a natural output because the minimal gauge-fixing can be defined on a flat background, where the $\mathcal{O}\left(\right.$ Riem $\left.^{3}\right)$ terms are irrelevant. Furthermore, the parameter $\alpha$ is only dependent on $\omega_{N, C}$. This is because only the term $C \square^{N} C$ in the action is responsible for the propagation of the spin-2 part of gravitational perturbations on flat spacetime with the highest number of derivatives.

The evaluation of the main expression

$\frac{i}{2} \ln \operatorname{Det} \hat{\mathcal{H}}=\frac{i}{2} \operatorname{Tr} \ln H^{\mu \nu, \rho \sigma}$

can be performed by using the generalized SchwingerDeWitt technique developed by Barvinsky and Vilkovisky [62], in a way qualitatively similar to the calculation of the cosmological constant counterterm in [43]. Technically, the calculation of the linear in $R$ term is much more complicated, and we shall describe it in certain details. The minimal choice of the gauge parameters (19) provides the useful form of the operator [43]

$H^{\mu \nu, \rho \sigma}=\mathcal{B}^{\mu \nu, \kappa \lambda} H_{\kappa \lambda}^{\prime}{ }^{\rho \sigma}$,

where $\mathcal{B}^{\mu \nu, \kappa \lambda}$ is the DeWitt metric in the space of the fields for our model,

$\mathcal{B}^{\mu \nu, \kappa \lambda}=\frac{\omega_{N, C}}{2}\left[\delta^{\mu \nu, \kappa \lambda}-\frac{\omega_{N, C}-6 \omega_{N, R}}{2\left(2 \omega_{N, C}-3 \omega_{N, R}\right)} g^{\mu \nu} g^{\kappa \lambda}\right]$,

where

$\delta^{\mu \nu, \kappa \lambda}=\frac{1}{2}\left(g^{\mu \kappa} g^{\nu \lambda}+g^{\mu \lambda} g^{\nu \kappa}\right)$

is the identity matrix in the space of the fields. In what follows we assume that the $c$-number operator $\mathcal{B}^{\mu \nu, \kappa \lambda}$ is nondegenerate, such that its inverse

$\mathcal{B}_{\alpha \beta, \mu \nu}^{-1}=\frac{2}{\omega_{N, C}}\left[\delta_{\alpha \beta, \mu \nu}+\frac{\omega_{N, C}-6 \omega_{N, R}}{18 \omega_{N, R}} g_{\alpha \beta} g_{\mu \nu}\right]$

has finite coefficients. Let us note that the degenerate case, when $\omega_{N, C}=0$ or $\omega_{N, R}=0$, is non-renormalizable, and therefore is much less interesting.

Acting on (21) with the inverse operator (23), one can see that the operator $H_{\kappa \lambda}^{\prime}{ }^{\rho \sigma}$ has the standard minimal form with the senior term of exactly $2 N+4$ derivatives, and with the identity matrix in the space of the fields

$\delta_{\kappa \lambda}^{\rho \sigma}=\frac{1}{2}\left(\delta_{\kappa}^{\rho} \delta_{\lambda}^{\sigma}+\delta_{\lambda}^{\rho} \delta_{\kappa}^{\sigma}\right)$

in front instead of the DeWitt metric $\mathcal{B}^{\mu \nu, \kappa \lambda}$ as in (21):

$$
\begin{aligned}
H_{\kappa \lambda}^{\prime} \rho \sigma= & \delta_{\kappa \lambda}^{\rho \sigma} \square^{N+2} \\
& +V_{\kappa \lambda}{ }^{\rho \sigma, \lambda_{1} \cdots \lambda_{2 N+2}} \nabla_{\lambda_{1}} \cdots \nabla_{\lambda_{2 N+2}} \\
& +W_{\kappa \lambda}{ }^{\rho \sigma, \lambda_{1} \cdots \lambda_{2 N+1}} \nabla_{\lambda_{1}} \cdots \nabla_{\lambda_{2 N+1}} \\
& +U_{\kappa \lambda}{ }^{\rho \sigma, \lambda_{1} \cdots \lambda_{2 N}} \nabla_{\lambda_{1}} \cdots \nabla_{\lambda_{2 N}}+\mathcal{O}\left(\nabla^{2 N-1}\right) .
\end{aligned}
$$

Obviously, the first factor in Eq. (21) does not contribute to the divergent part of the effective action, and therefore for us 
$\frac{i}{2} \operatorname{Tr} \ln H^{\mu \nu, \rho \sigma}=\frac{i}{2} \operatorname{Tr} \ln H_{\kappa \lambda}^{\prime} \rho \sigma$.

Different from the calculation of the cosmological constant counterterm [43], the expressions for $U$ and $V$ requested for the derivation of divergences for an arbitrary metric background are very bulky and difficult to derive and to deal with. However, there are possibilities of great simplifications when we are interested only in the counterterm linear in scalar curvature. First of all, by dimensional reasons the mass dimensions of $V, W$ and $U$ are 2, 3 and 4, correspondingly. If only coefficients $\omega_{N, i}(i=R, C, \mathrm{~GB})$ entered the expression for $H^{\prime}$ tensor, then only $U$ could be relevant in our case. However, the other coefficients $\omega_{N-1, i}$ enter too, hence both $V$ and $U$ parts are relevant here. Furthermore, since we do not pretend to calculate the $\square R$-term, in the course of calculations we can assume that the scalar curvature $R$ is a constant, without losing the generality. Finally, it is good to remember that the Ricci and Riemann tensors enter the final expressions for the linear in scalar curvature term only through their contractions with the metric tensor. Therefore, again without loss of generality we can assume that the curvature tensor is

$R_{\mu \nu \alpha \beta}=\frac{1}{3} \Lambda\left(g_{\mu \alpha} g_{\nu \beta}-g_{\nu \alpha} g_{\mu \beta}\right)$,

where $\Lambda$ is a constant. This is equivalent of assuming that the background is a maximally symmetric spacetime (dS or AdS) with the radius of curvature given by $\pm \sqrt{3 / \Lambda}$. Due to the covariant constancy of the purely background metric we have $W=0$. At the end one can set $\Lambda=R / 4$ and arrive at the general result of the counterterm in the one-loop effective action, which is valid for both constant and nonconstant scalar curvature $R$, in the given approximation. On the basis of dimensional analysis, in the tensor $V$ there are terms of zero and first powers in the background curvature $\Lambda$, that can be written schematically as

$V=\tilde{V}_{(0)}+\Lambda \tilde{V}_{(1)}$,

while for the tensor $U$ there are terms of the following types

$U=\tilde{U}_{(0)}+\Lambda \tilde{U}_{(1)}+\Lambda^{2} \tilde{U}_{(2)}$

that means up to the second power in background curvature. The dimensions of the different types of terms are compensated by the coefficients, which may have different powers of dimensional parameters of the action (2), e.g. by the ratios $\omega_{N-1, i} / \omega_{N, j}$, where $i, j=R, C$, or GB. The details of this structure can be found in the Appendix A.

In order to use the generalized Schwinger-DeWitt technique of [62], we can first extract the highest derivatives in the form of $\square^{N+2}[43]$,

$$
\begin{aligned}
\operatorname{Tr} \ln H_{\kappa \lambda}^{\prime}{ }^{\rho \sigma}= & \operatorname{Tr} \ln \square^{N+2}+\operatorname{Tr} \ln \left\{\delta_{\kappa \lambda}^{\rho \sigma}\right. \\
& +V_{\kappa \lambda}{ }^{\rho \sigma, \alpha_{1} \cdots \alpha_{2 N+2}} \nabla_{\alpha_{1}} \cdots \nabla_{\alpha_{2 N+2}} \frac{1}{\square^{N+2}} \\
& +W_{\kappa \lambda}{ }^{\rho \sigma, \alpha_{1} \cdots \alpha_{2 N+1}} \nabla_{\alpha_{1}} \cdots \nabla_{\alpha_{2 N+1}} \frac{1}{\square^{N+2}} \\
& +U_{\kappa \lambda}{ }^{\rho \sigma, \alpha_{1} \cdots \alpha_{2 N}} \nabla_{\alpha_{1}} \cdots \nabla_{\alpha_{2 N}} \frac{1}{\square^{N+2}} \\
& \left.+\mathcal{O}\left(\nabla^{2 N-1} \frac{1}{\square^{N+2}}\right)\right\}
\end{aligned}
$$

and after that perform a series expansion of the logarithm. The first term in the last expression does not produce linear in curvature divergences and the last term includes terms that cannot give relevant contributions by dimensional reasons. In what follows we omit the last term. The expression can be further elaborated as follows, reducing to the universal traces of [62],

$$
\begin{aligned}
& \operatorname{Tr} \ln H_{\kappa \lambda}^{\prime}{ }^{\rho \sigma}=(N+2) \operatorname{Tr} \ln \square \\
& +\operatorname{Tr}\left\{V_{\kappa \lambda}{ }^{\rho \sigma, \alpha_{1} \cdots \alpha_{2 N+2}} \nabla_{\alpha_{1}} \cdots \nabla_{\alpha_{2 N+2}} \frac{1}{\square^{N+2}}\right\} \\
& +\operatorname{Tr}\left\{W_{\kappa \lambda}{ }^{\rho \sigma, \alpha_{1} \cdots \alpha_{2 N+1}} \nabla_{\alpha_{1}} \cdots \nabla_{\alpha_{2 N+1}} \frac{1}{\square^{N+2}}\right\} \\
& +\operatorname{Tr}\left\{U_{\kappa \lambda}{ }^{\rho \sigma, \alpha_{1} \cdots \alpha_{2 N}} \nabla_{\alpha_{1}} \cdots \nabla_{\alpha_{2 N}} \frac{1}{\square^{N+2}}\right\} \\
& -\frac{1}{2} \operatorname{Tr}\left\{V_{\kappa \lambda}^{\mu \nu, \alpha_{1} \cdots \alpha_{2 N+2}} V_{\mu \nu}{ }^{\rho \sigma, \beta_{1} \cdots \beta_{2 N+2}}\right. \\
& \left.\times \nabla_{\alpha_{1}} \cdots \nabla_{\alpha_{2 N+2}} \nabla_{\beta_{1}} \cdots \nabla_{\beta_{2 N+2}} \frac{1}{\square^{2 N+4}}\right\} .
\end{aligned}
$$

Let us start the analysis of the last expression with two simple observations. The commutators of covariant derivatives with $V$ and $\square$ to negative power operators in the last term were disregarded because they are irrelevant in the linear in curvature approximation, as it was explained above. According to the Eq. (30) and the table of universal traces of [62], the trace of the operator proportional to the tensor $W$ vanishes identically, which confirms our previous consideration. Therefore, we need to consider only linear in $V$ and $U$ single traces and the quadratic in $V$ mixed (interference) trace. Due to the fact that in the tensors $V$ and $U$ we have at most four free covariant derivatives (not contracted with other derivatives) we can simplify the relevant parts of the expression (30) to the form

$$
\begin{aligned}
\operatorname{Tr} \ln H_{\kappa \lambda}^{\prime}{ }^{\rho \sigma}= & \operatorname{Tr}\left\{V_{\kappa \lambda}{ }^{\rho \sigma, \alpha \beta \gamma \delta} \nabla_{\alpha} \nabla_{\beta} \nabla_{\gamma} \nabla_{\delta} \frac{1}{\square^{3}}\right\} \\
& +\operatorname{Tr}\left\{U_{\kappa \lambda}{ }^{\rho \sigma, \alpha \beta \gamma \delta} \nabla_{\alpha} \nabla_{\beta} \nabla_{\gamma} \nabla_{\delta} \frac{1}{\square^{4}}\right\}
\end{aligned}
$$




$$
\begin{aligned}
& -\frac{1}{2} \operatorname{Tr}\left\{V_{\kappa \lambda}{ }^{\mu \nu, \alpha_{1} \beta_{1} \gamma_{1} \delta_{1}} V_{\mu \nu}{ }^{\rho \sigma, \alpha_{2} \beta_{2} \gamma_{2} \delta_{2}}\right. \\
& \left.\times \nabla_{\alpha_{1}} \nabla_{\beta_{1}} \nabla_{\gamma_{1}} \nabla_{\delta_{1}} \nabla_{\alpha_{2}} \nabla_{\beta_{2}} \nabla_{\gamma_{2}} \nabla_{\delta_{2}} \frac{1}{\square^{6}}\right\}+\cdots,
\end{aligned}
$$

where we omitted terms which do not contribute to the divergences linear in $R$. The explicit expressions for the $c$-number tensors $U$ and $V$ can be found in Appendix A.

We here make some comments on the correct way of taking the traces that are in general not an easy task. It is important to note that the expression above includes all the divergences that are linear in $R$. A special care must be given to the interference term $-\frac{1}{2} \operatorname{Tr} V^{2}$. Due to dimensional reasons we are interested in the mixing terms only between the parts of the $V$ tensor that are linear in $\Lambda$ or independent on $\Lambda$. Only these terms can give the correct dimension of the coefficient of $R$ as well as the correct power of background curvature. Then for the mixing terms we do not need to account for additional background curvature arising from commutations. Furthermore, when taking the single trace of $V$ we need only terms independent on $\Lambda$. Contrary, in the traces that involve the tensor $U$ we are interested only in those parts that are proportional to $\Lambda$.

The final technical comments are the following. The interesting parts, which give divergent contributions linear in curvature, of $V$ and $U$ are linearly proportional to the couplings $\omega_{N-1, C}, \omega_{N-1, R}$ or $\omega_{N-1, \mathrm{~GB}}$ and originate from the variation of the corresponding part of the action (2). These contributions must be independent on $\Lambda$ in the $V$ part and linear in $\Lambda$ in the $U$ part, while the denominators with $\omega_{N, R}$ or $\omega_{N, C}$ emerge only because of the inverse DeWitt metric (23). To derive the relevant $U$ term correctly one has to take into account the commutations up to the order linear in background curvature in the second variation of the gravitational action (2).

The situation with the interference term is different. The first interference factor in $V$ has the same origin as in the single trace discussed above. However, the second one must be precisely linear in $\Lambda$ and independent on the couplings $\omega_{N-1, i}$. It contains some rational functions of the dimensionless ratios between the coefficients $\omega_{N, R}, \omega_{N, C}$ and $\omega_{N, \mathrm{~GB}}$. The origin of this term is either from the second variation of the gravitational action or from the gauge-fixing term, both multiplied by the inverse DeWitt metric. To derive this term correctly one has to take into account the commutations up to the order linear in background curvature both in the second variation of the gravitational action or the gauge-fixing term. In the intermediate expressions one can observe rather complicated rational functions, and only in the final traces there are essential simplifications. The precise expressions for relevant parts of tensors $V$ and $U$ are not given here due to their lengths.
Actually in (31) we will never need to take universal trace with more than four free covariant derivatives because of the structure of the indices and because only the metric functions appear in the $V$ and $U$ tensors. Therefore, the last but one term in (31) will never be so long after explicit contractions.

\subsection{Divergences for $N \geqslant 2$ models}

After taking the traces according to [62], we can write the results for the divergent part of the one-loop effective action. The part linear in curvature is given by the expression

$$
\begin{aligned}
& \Gamma_{\mathrm{EH}, \mathrm{div}}^{(1)}(N, \mathrm{~GB})=\frac{\mu^{n-4}}{2(4 \pi)^{2}} \frac{1}{\varepsilon} \int d^{n} x \sqrt{|g|} \\
& \quad \times\left\{\frac{5 \omega_{N-1, C}}{6 \omega_{N, C}}+\frac{\omega_{N-1, R}}{6 \omega_{N, R}}\right. \\
& +(2 N-1)\left[\frac{5 \omega_{N-1, C}}{6 \omega_{N, C}^{2}}-\frac{\omega_{N-1, R}}{18 \omega_{N, R}^{2}}\right] \omega_{N, \mathrm{~GB}} \\
& \left.+(2 N-3)\left[\frac{5}{6 \omega_{N, C}}-\frac{1}{18 \omega_{N, R}}\right] \omega_{N-1, \mathrm{~GB}}\right\} R,
\end{aligned}
$$

where $\varepsilon=(4-n) / 2$ is the parameter of dimensional regularization, $\mu$ is the dimensionful regularization parameter and by $n$ we denote spacetime dimensionality. One can see that the generalized Gauss-Bonnet terms with the coefficients $\omega_{N, \mathrm{~GB}}$ and $\omega_{N-1, \mathrm{~GB}}$ give non-zero contributions in the formulas above, differently from the four-derivative quantum gravity case $[12,15]$.

Let us stress that the result for the divergence (33) is valid only for $N \geqslant 2$ and there is no smooth limit $N \rightarrow 0$ or even $N \rightarrow 1$. The reason for this discontinuity is that the expansion of $\square$ into the quantum field $h_{\mu \nu}$ generates expressions of the $\square^{-1}$ and $\square^{-2}$ type, which are badly defined when acting on a constant. As a result it becomes impossible to take the limits $N \rightarrow 0$ and $N \rightarrow 1$ in the bilinear form of the generalized Gauss-Bonnet term. After this expansion the formulas do not "recognize" that for $N=0$ this term is topological. This is why the coefficients of the $\omega_{N-1, \mathrm{~GB}}$ and $\omega_{N, \mathrm{~GB}}$ terms are proportional to $2 N-1$ and $2 N-3$, and not to $N$, as one might have expected from them. Indeed, the same discontinuity takes place also for the cosmological constant - type divergence derived in [43]. We have checked independently that for $N=0$ the Gauss-Bonnet term does not contribute to the divergences. The details are not shown here, because one can find them in the previous works $[12,15]$. We also remark that even for the calculations in highly nonlocal theory with the expressions of the $\square^{-1}$ and $\square^{-2}$ type for one-loop divergences one is not required to impose proper boundary conditions for these operators. This detail has been known from long time ago, e.g., from the pioneer paper by Ichinose [63]. 
If the relevant generalized Gauss-Bonnet terms are absent, $\omega_{N-1, \mathrm{~GB}}=0$ and $\omega_{N, \mathrm{~GB}}=0$, we arrive at a very compact form of divergences, which is valid for $N \geqslant 2$,

$$
\begin{aligned}
\Gamma_{\mathrm{EH}, \mathrm{div}}^{(1)}= & \frac{\mu^{n-4}}{12(4 \pi)^{2} \varepsilon} \int d^{n} x \sqrt{|g|}\left\{\frac{5 \omega_{N-1, C}}{\omega_{N, C}}\right. \\
& \left.+\frac{\omega_{N-1, R}}{\omega_{N, R}}\right\} R \equiv-\frac{\mu^{n-4}}{2 \varepsilon} \int d^{4} x \sqrt{|g|} \beta_{G} R .
\end{aligned}
$$

One can note that in the last expression the number of derivatives $N$ does not appear explicitly, but only through the coefficients of the action (2). This is in contrast to the more general formula (33), where the explicit dependence on $N$ takes place in the sector related to the generalized Gauss-Bonnet terms.

For the sake of completeness, let us write down the expression for the cosmological constant divergences, derived in [43], in the notations which we use here,

$$
\begin{aligned}
\Gamma_{\mathrm{cc}, \mathrm{div}}^{(1)}= & -\frac{\mu^{n-4}}{2(4 \pi)^{2}} \frac{1}{\varepsilon} \int d^{n} x \sqrt{|g|}\left(\frac{5 \omega_{N-2, C}}{\omega_{N, C}}\right. \\
& \left.+\frac{\omega_{N-2, R}}{\omega_{N, R}}-\frac{5 \omega_{N-1, C}^{2}}{2 \omega_{N, C}^{2}}-\frac{\omega_{N-1, R}^{2}}{2 \omega_{N, R}^{2}}\right) \\
\equiv & -\frac{\mu^{n-4}}{2 \varepsilon} \int d^{4} x \sqrt{|g|} \beta_{\Lambda_{\mathrm{cc}}} .
\end{aligned}
$$

The contribution of the generalized GB term to the cosmological constant divergence is zero because this term does not contribute to the propagator around flat spacetime. It is useful to remember that both expressions (33) and (34) are valid only for $N \geqslant 2$. It is easy to see that the relative factor of " 5 " between the contributions of tensor and scalar modes in Eq. (33) is exactly the same which one could observe in the cosmological constant divergence (34).

It is clear from Eq. (33) that one can provide zero divergence in the Einstein-Hilbert sector by adjusting the highestderivative coefficients of the classical action $\omega_{N-1, C}, \omega_{N, C}$, $\omega_{N-1, R}$ and $\omega_{N, R}$. This can be achieved even without using the freedom to choose the coefficients of the $\omega_{N-1, \mathrm{~GB}}$ and $\omega_{N, \mathrm{~GB}}$ in (33). Moreover, one can make the same for both the cosmological and the linear in $R$ divergences at the same time.

One can make zero the two remaining relevant coefficients for the $C^{2}$ and $R^{2}$ counterterms by means of the "killer" operators. Thus one can achieve the one-loop finiteness, which can be directly extended into all-loop order for the case of $N \geqslant 3$, since in this case divergences exist only at the oneloop level.

\subsection{The special cases of $N=1$ and $N=0$}

In order to achieve a better understanding of the situation with smaller $N$, let us present the corresponding results, without going into full details. As we have explained above, the cases of $N=0$ and $N=1$ should be considered separately. The calculations are pretty much the same as for $N \geqslant 2$, therefore, we present only the final results.

For $N=1$ the action includes the following relevant terms:

$$
\begin{aligned}
S_{N=1}= & \int d^{4} x \sqrt{|g|}\left\{\omega_{1, R} R \square R+\omega_{1, C} C \square C\right. \\
& \left.+\omega_{1, \mathrm{~GB}} \mathrm{~GB}_{1}+\omega_{0, R} R^{2}+\omega_{0, C} C^{2}\right\} .
\end{aligned}
$$

The term linear in curvature in the divergent part of the oneloop effective action is:

$$
\begin{aligned}
\Gamma_{\mathrm{div}}^{(1)}= & -\frac{\mu^{n-4}}{2(4 \pi)^{2}} \frac{1}{\varepsilon} \int d^{n} x \sqrt{|g|}(-1)\left\{\frac{5 \omega_{0, C}}{6 \omega_{1, C}}+\frac{\omega_{0, R}}{2 \omega_{1, R}}\right. \\
& \left.-\frac{5 \omega_{0, R}}{\omega_{1, C}}+\left(\frac{5 \omega_{0, C}}{6 \omega_{1, C}^{2}}-\frac{\omega_{0, R}}{18 \omega_{1, R}^{2}}\right) \omega_{1, \mathrm{~GB}}\right\} R .
\end{aligned}
$$

One can easily note that this expression differs from the $N \geqslant 2$ case. In particular, there is a mixing of the coefficients for the Weyl and scalar curvature. Moreover, we notice that only the terms with the Weyl curvatures and with the generalized Gauss-Bonnet term give rise to divergences that can be obtained continuously from formula (33) in the limit $N=1$.

For $N=0$ the action reduces to the fourth-order quantum gravity, which was the subject of similar considerations in [7-12], namely

$S_{N=0}=\int d^{4} x \sqrt{|g|}\left\{\omega_{R} R^{2}+\omega_{C} C^{2}+\omega_{\mathrm{EH}} R\right\}$.

We performed the corresponding one-loop calculation just to make an extra check. The linear in curvature divergent part of the one-loop effective action is given by

$$
\begin{aligned}
\Gamma_{\mathrm{div}}= & -\frac{\mu^{n-4}}{2(4 \pi)^{2} \varepsilon} \int d^{n} x \sqrt{|g|}\left\{\frac{1}{12 \omega_{R}}\right. \\
& \left.-\frac{13}{12 \omega_{C}}-\frac{5 \omega_{R}}{2 \omega_{C}^{2}}\right\} \omega_{\mathrm{EH}} R .
\end{aligned}
$$

Taking into account the difference in notations, this expression perfectly agrees with the well-known result of [9-11]. Let us remember, once again, that this expression depends on the gauge-fixing [8]. The expression (38) corresponds to the minimal gauge that is the simplest one for the sake of practical calculations.

Let us present more detailed explanation for the above mentioned discontinuity between $N=0, N=1$ and $N \geqslant 2$ results. It is worth noticing that there exists another explanation which is related to the action of the $\square^{-1}$ operator [64]. We leave it to the interested reader to consult this work, and we present here the explanation which is purely technical. The qualitative reason for the discontinuity is as follows. In the course of computations of the second variation of the action on a general background one has to take into account 
variations of the $\square$ operator and its powers. And here is the source of the discontinuity. When $N=0$, (that means no $\square$ operators) there is no variation of these operators. When $N=1$ there are variations of only one $\square$ operator. There may be first or second variations, but always of one $\square$. Starting from $N=2$ there are qualitatively new terms with two first variations of two different $\square$ operators appearing in the factor expansion of the power $\square^{N}$. We also have to take into account that these terms, which matter for computation of divergences proportional to the Ricci scalar $R$, are from level $N-1$, while the exponent on the box operator leading in UV is $N$, according to (2).

These structural differences in taking variations are the reason for discontinuities between $N=0, N=1$, and $N=$ 2 cases, which are present in the vertices of the theory, or in the expressions for $V, W$ and $U$ tensors. However, these differences do not occur in the leading terms in (25), which correspond to the propagator on a flat spacetime background.

Let us note that the structure of vertices explained above is well presented in the thesis [65] for the perturbative calculus around flat gauge backgrounds and using Feynman technique. The theory presented there is with action quadratic in field strengths and containing a form factor of the covariant $\square$ operators. As we know from [43] for the beta function of the cosmological constant we are interested only in the propagator on flat spacetime, so there the variations of the $\square$ are not producing the effect of discontinuity. This effect shows up in the divergences of the $R$-type which we derived here, and is expected in the four-derivative divergences as well which are yet to be calculated. The reason for discontinuities for the beta function of the cosmological constant case is due to structural changes of the action. Namely the Einstein-Hilbert term $R$ is neither of the form $R \square^{N} R$ nor $C \square^{N} C$ for none $N$, neither for $N$ analytically continued to a domain of negative integers.

\section{Quantum nonlocal models}

It is tempting to use the results for the divergences in the polynomial theory (33), (33), (34) in order to obtain the same type of divergences in a wide class of the nonlocal models of quantum gravity. The most interesting theories are certainly the ones that have no massive ghost-like poles in the propagators. These models were introduced by Tseytlin [66] in the framework of string theory as an alternative to the Zwiebach transformation of metric [67-69] in the low-energy effective action. Another interesting feature of the original nonlocal model [66] was the absence of singularity in the Newtonian solution for the gravitational field of a point-like massive particle. A ghost-free nonlocal gravitational action was proposed for the first time by Krasnikov in 1988 [44] and studied by Kuz'min in 1989 [45]. Later on, qualitatively similar ghost- free nonlocal models were suggested by Tomboulis [70] as candidates to be unitary and super-renormalizable or even finite quantum gravity theories [54-58]. One can remember that the general aspects of quantization of nonlocal quantum field theories have a long history [71]. For the recent developments in many aspects of nonlocal theories the reader can follow the literature in [54-58,72-82] about the localization of nonlocal theories and nonlocal gravity as a diffusion equation. Recently in the paper of one of the present authors [59] it was shown that an infinite amount of the ghost-like complex states in these models emerge in the quantum theory, when one takes the loop corrections into account. However, perturbative unitarity of the $S$-matrix is guaranteed by the Cutkosky rules that are not modified by the kind of non locality that we are considering here. Nevertheless, it is important to have a better understanding of the possible form of quantum corrections in such theories. Let us start the discussion of this problem from the simplest example [66].

Our starting point will be the nonlocal theory of quantum gravity with the classical action $[44,45,54-56,70]$

$$
\begin{aligned}
S_{\mathrm{nl}}= & \int d^{4} x \sqrt{|g|}\left\{-\frac{1}{\kappa^{2}} R\right. \\
& \left.+\frac{1}{2} C \Phi\left(\frac{\square}{M^{2}}\right) C-\frac{1}{6} R \Psi\left(\frac{\square}{M^{2}}\right) R\right\} .
\end{aligned}
$$

We do not add a possible nonlocal generalization of the Gauss-Bonnet term, but this is possible. The power counting in this theory reduces to the topological relation (7) between the number of loops, internal lines and vertices [59]. Its evaluation shows the result may be identical to the one of the polynomial theory (2) with $N \geqslant 3$. This means that the divergences emerge only at the one-loop order and have the form of the cosmological constant and Einstein-Hilbert terms, plus two relevant terms with four derivatives, namely $C^{2}$ and $R^{2}$. In order to achieve this, one has to choose $\Phi(z)$ and $\Psi(z)$ functions to have equal asymptotic behaviour and be sufficiently fast growing functions at $z \rightarrow \infty$. It is clear that we have to deal only with the model (2) with a very large $N$, hence the result for the cosmological and linear in $R$ divergences in the polynomial theory are given by the expressions (34) and (33).

The most natural option is to choose $\Psi$ and $\Phi$ such that the structures of the Euclidean propagator in spin-2 and spin0 sectors are the same. Consider the simplest version of the theory [83-85],

$\Phi=\Psi=-\frac{1}{\kappa^{2} \square}\left(e^{-\square / M^{2}}-1\right)$.

Then we arrive at the equation for the pole

$1+\kappa^{2} p^{2} \Phi\left(-\frac{p^{2}}{M^{2}}\right)=e^{p^{2} / M^{2}}$, 
such that the unique spin-2 pole of the propagator is massless. In what follows we consider only this version of the theory, but it is certainly possible to make generalizations.

Let us show, by taking a limit $N \rightarrow \infty$ in the results (33), (33) and (34), that the coefficients of the one-loop divergences in the model (39) with functions (41) experience an unrestricted growth. In the polynomial theory the equation for the poles of the propagator is defined by

$1+\kappa^{2} p^{2} \Phi\left(-\frac{p^{2}}{M^{2}}\right)=\sum_{k=1}^{N} \frac{\left(p^{2} / M^{2}\right)^{k}}{k !}$

and the same for the function $\Psi$. Then the relevant coefficients of the polynomial action (2) have the structure

$\left(\omega_{k, C} ; \omega_{k, R}\right) \sim \frac{M^{-2 k}}{k !}, \quad k=N, N-1, N-2$.

The inspection of the coefficients of expressions (33) and (34) shows that they behave like $N$ and $N^{2}$, correspondingly. In the limit $N \rightarrow \infty$ both coefficients explode, that demonstrates a discontinuity of quantum corrections for the exponential theory in the given approach. This shows that in genuinely nonlocal theories a new type of one-loop divergences appear (divergences of large $N$ ). Let us note that one can provide the finiteness of the theory for each $N$ in a way we described above, and then the limit $N \rightarrow \infty$ is well-defined. For the contributions to the inverse of Newton constant this may be achieved by adjusting the $\omega_{N-1, \mathrm{~GB}}$ and/or $\omega_{N, \mathrm{~GB}}$ coefficients to provide zero result for all $N$ in Eq. (33). These coefficients would explicitly depend on $N$. For sensible nonlocal theory defined in the limit $N \rightarrow \infty$ these coefficients should survive, but the explicit evaluation of them shows that they are decaying like $1 / N$. In conclusion such generalized Gauss-Bonnet terms are not present in the final action in the limit $N \rightarrow \infty$ of the nonlocal theory. One may try to add standard killer operators [57]. However, without explicit calculations it is unclear whether this will be sufficient to provide an UV-finite nonlocal theory.

The expressions of the type $\omega_{N-1, i} / \omega_{N, i}$ appear in (33), where there is no mixing term. In full generality the diagonality in $i, j$ indices $(i, j=R$ or $C$ ) lets us to interpret such ratio in the limit $N \rightarrow \infty$ as a d'Alembert definition of the convergence radius ${ }^{2}$ of the complex function defined by the series $f_{i}(z)=\sum_{n=0}^{\infty} \omega_{n, i} z^{n}$, that is

$\rho_{f_{i}}=\lim _{N \rightarrow \infty} \frac{\omega_{N-1, i}}{\omega_{N, i}}$.

This result can be exported also for the similar interpretation of the divergence proportional to the cosmological constant. From this it is obvious that if any form factor like $\Phi$ or $\Psi$ is an entire function on the complex plane, then the new

\footnotetext{
2 Precisely speaking this is a convergence radius of a Taylor series expansion around the origin $z=0$.
}

type of divergence will inevitably appear. However, this formula gives also the possibility of defining the standard divergences for theories in which $\Phi$ or $\Psi$ are other non-analytic real functions.

In the first part of this section we studied the simpler example of nonlocality that turns out to be sufficient to make convergent scalar field theories in Euclidean signature. In Minkowskian signature it is sufficient to replace $\exp \left(-\square / M^{2}\right)$ by $\exp P\left(\square / M^{2}\right)$, where $P\left(\square / M^{2}\right)$ is a polynomial of even degree in its argument. However, in a gravitational or a non-abelian gauge theory case such nonlocality seems not suitable exactly because of the gauge symmetry that forces the kinetic and interaction operators to have the same ultraviolet scaling, which implies nonlocal divergences. However, we can overcome this issue with a special class of nonlocal form factors that enjoy the property to be asymptotically polynomial in a region around the real axis $[45,54-57,70]$.

Let us give here an explicit example of such function which is supposed to replace the exponential $\exp \left(-\square / M^{2}\right)$ in (40),

$e^{H(z)}=e^{\frac{1}{2}\left[\Gamma\left(0, p(z)^{2}\right)+\gamma_{E}+\ln \left(p(z)^{2}\right)\right]}$ for $z=\square$,

where $p(z)$ is a polynomial of degree $N, \gamma_{E}$ is the EulerMascheroni constant and finally $\Gamma(a, x)$ is the incomplete Gamma function. The crucial property of the function in (45) is that the divergent contributions to the beta functions only depend on the local asymptotic polynomial $p(z)$, while the full nonlocality manifests itself only in the finite contributions to the quantum effective action. It is easy to see that any correction to the UV polynomial is exponentially suppressed [57]. Therefore, all results which were reported for the polynomial case can be applied to this class of theories.

Let us note that the beta functions in the case under consideration are one-loop exact and that two out of the total four relevant divergent contributions to the quantum effective action are known. The last observation is that in the paper [45] it is stated that the beta functions for $R^{2}$ and $R_{\mu \nu}^{2}$ vanish, but we are unable to confirm or verify this statement.

All the beta functions for local or nonlocal theories have been computed using the heat-kernel expansion and Barvinsky-Vilkovisky trace technology, therefore, in Euclidean space. The analytic continuation to the Minkowski space can be implemented on the basis of recent developments. We will deal with this subject further in a future work.

\section{Renormalization group equations}

Let us come back to the polynomial theory (2) and consider the case without killer terms, when the divergences are given by the expressions (33) and (34). In this case one can con- 
struct the renormalization group equations for the parameters $\omega_{\mathrm{EH}}$ and $\omega_{\mathrm{cc}}$ of the classical action. The derivation of these equations is almost a trivial task (see, e.g., [61] for the introduction), but for the sake of completeness we provide the explanations here. The system of renormalization group equations for the running coupling constants is defined by relations

$\frac{d \alpha_{i}}{d t}=\beta_{i}, \quad$ with $\quad t=\ln \frac{\mu}{\mu_{0}}$.

Here $\alpha_{i}(t)$ are obtained by making the coupling constants $\alpha_{i}$ scale-dependent in the classical action. The renormalized Lagrangian is

$$
\begin{aligned}
\mathcal{L}_{\text {ren }}= & \mathcal{L}\left(\alpha_{i}(t)\right)+\mathcal{L}_{\mathrm{ct}}=\mathcal{L}\left(\alpha_{i}(t)\right)-\left(Z_{\Lambda_{\mathrm{cc}}}-1\right) \frac{2 \Lambda_{\mathrm{cc}}}{16 \pi G} \\
& -\left(Z_{G}-1\right) \frac{1}{16 \pi G} R+\cdots,
\end{aligned}
$$

where $\mathcal{L}_{\mathrm{ct}}$ is a counterterm and $Z_{\alpha_{i}}$ denote renormalization constant of the couplings $\alpha_{i}$. Let us refer to the further details in Appendix B.

The final results for the beta functions for $N \geqslant 2$ are as follows:

$$
\begin{aligned}
\beta_{G} & =\mu \frac{d}{d \mu}\left(-\frac{1}{16 \pi G}\right) \\
& =-\frac{1}{6(4 \pi)^{2}}\left(\frac{5 \omega_{N-1, C}}{\omega_{N, C}}+\frac{\omega_{N-1, R}}{\omega_{N, R}}\right), \\
\beta_{\mathrm{cc}} & =\mu \frac{d}{d \mu}\left(-\frac{\Lambda_{\mathrm{cc}}}{8 \pi G}\right) \\
& =\frac{1}{(4 \pi)^{2}}\left(\frac{5 \omega_{N-2, C}}{\omega_{N, C}}+\frac{\omega_{N-2, R}}{\omega_{N, R}}-\frac{5 \omega_{N-1, C}^{2}}{2 \omega_{N, C}^{2}}-\frac{\omega_{N-1, R}^{2}}{2 \omega_{N, R}^{2}}\right) .
\end{aligned}
$$

A pertinent observation is that for $N \geqslant 3$ these two beta functions are exact, since higher loops would not provide further contributions. The next question is that whether these beta functions have physical sense. In other words, we have to describe the situation when they may describe the running of the gravitational and cosmological constants.

The physical sense of the running of the inverse Newton and cosmological constant is a complicated problem. It is known [64] (see also [87,88]) that such a running does not correspond to the non-local $\square$-dependent form factors in the loop corrections to the classical action. The reason is that in both cases such a form factors would act on a constant or produce a series of irrelevant (without boundaries) surface terms. These arguments mean that the running of $G$ and $\Lambda$ cannot be understood in a usual Quantum Field Theory sense, which is based on the scattering amplitudes on the flat background. At the same time this does not forbid the running, because physically interesting gravitational observables do not reduce to such amplitudes. The reader can consult Refs. $[28,86]$ for further references on this interesting issue, in the part of the cosmological constant running. Also, a kind of a regular procedure of the physically relevant scale identification [29] for the running of Newton constant was discussed in [89] and some other works.

It is instructive to compare (49) with the general arguments based on covariance and dimensional considerations, Eq. (1). For this end we have to identify what are the masses in the theory under consideration. In general, the theory of higher derivative quantum gravity (2) possesses many massive degrees of freedom, some of them ghost-like and some normal [43]. Then our Eq. (49) is exactly (1), where the dimensionless ratios between different $\omega$ 's play the role of coupling constants.

The most interesting is the case when all massive states have complex masses $m_{i}$. The theory may be consistent only if these masses enter as complex conjugate pairs. This condition is provided by a real classical action of the theory. But the same condition applies also to the renormalization group improved action. Therefore, in this case Eq. (1) boils down to the reduced version

$\mu \frac{d}{d \mu} \frac{1}{G}=\sum_{i} \alpha_{i} m_{i}^{*} m_{i}=\sum_{i} \alpha_{i}\left|m_{i}\right|^{2}$,

where $\alpha_{i}$ are real coefficients depending on the dimensionless ratios of the coefficients $\omega$.

As it was extensively discussed in [28] and in the review paper [86], it is not clear that both or any of the renormalization group equations can be applied to cosmology and astrophysics. The reason is that the diagrams which lead to Eqs. (49) and (50) include internal lines of a massless graviton, but also of the number of massive states. In the most interesting cases of unitary models of higher derivative quantum gravity these massive poles are all complex [46,47]. Then we face an unsolved problem of what remains from the effects of quantum gravity with massive modes at low energy [12]. It is well-known that the contributions of the loops of massive fields do decouple in the IR, in accordance with the gravitational version of the Appelquist and Carazzone theorem $[87,88]$. However, this is a well-established result only for the contributions of massive matter fields, when all internal lines of the diagrams have the propagators with equal masses. In the case of quantum gravity one has to deal with a more complicated case of mixed loops, where some of the internal lines are massive and some are massless, or have much lighter mass. Needless to say that the situation becomes much more tricky in the case of numerous complex masses. The analysis of this issue is certainly very interesting, but it is beyond the scope of the present work. In any case the expressions (49) and (50) represent a good starting point, being a universal UV limits for the physical beta functions under discussion. 


\section{Conclusions}

The one-loop calculations always represent one of the most important elements in understanding new theories of quantum gravity. These calculations are especially difficult in the case of higher derivative models. In the present work, the one-loop calculations in the super-renormalizable quantum gravity theory has been extended to the term linear in scalar curvature. Together with the previous derivation [43] in the cosmological constant sector, this enables us to obtain the closed system of renormalization group equations (49) and (50), which may be a basis for establishing the physically relevant running of Newton and cosmological constants in both high- and low-energy regimes.

In our opinion, the following two aspects of superrenormalizable quantum gravity theories make the result especially relevant. First of all, in the models with $N \geqslant 2$ the one-loop beta functions are exact. Therefore, in (49) and (50) we have the first example of exact beta functions in four-dimensional quantum gravity. Second, some versions of these theories have only complex conjugate pairs of massive ghost-like states, which turn out to be unitary at tree-level [46,47] and at perturbative level [48]. The results obtained here cover these cases and, therefore, can be seen as a first example of beta functions in a class of consistent models of perturbative quantum gravity.

One of the most remarkable features of the result (49) is its universality. For the models with $N \geqslant 3$ this is the first example of an exact beta function for the Newton constant ever calculated in quantum gravity models. Needless to say that there are many approaches that derive the non-perturbative renormalization group flows in different models of quantum gravity. The expressions (49) and (50) can be used as a test for these approaches - something that cannot be done with (50) alone.

Last but not least, an important aspect concerning the beta functions (49) and (50) is that they are based on the Minimal Subtraction scheme of renormalization, and, therefore, correspond to the running of $G^{-1}$ and $\rho_{\Lambda}$ in the UV. The most interesting running of these parameters is the one in the IR, and this subject opens a vast area for the future work, where the universal expressions $G^{-1}$ and $\rho_{\Lambda}$ may be used as tester for different approaches to the physical running and consequent applications in cosmology and astrophysics.

Acknowledgements This work has been partially started during the visit of I.Sh. to the group of Theoretical Physics in Fudan University. The kind hospitality of this group is gratefully acknowledged. I.Sh. is thankful to CNPq, FAPEMIG and ICTP for partial support of his work. Part of the preparation of the manuscript by I.Sh. was done during the workshop in Mainz, and I.Sh. is grateful to the Mainz Institute for Theoretical Physics (MITP) for the hospitality and partial support of his work during this event.
Open Access This article is distributed under the terms of the Creative Commons Attribution 4.0 International License (http://creativecomm ons.org/licenses/by/4.0/), which permits unrestricted use, distribution, and reproduction in any medium, provided you give appropriate credit to the original author(s) and the source, provide a link to the Creative Commons license, and indicate if changes were made.

Funded by $\mathrm{SCOAP}^{3}$.

\section{Appendix A}

We here collect the list of bilinear expansions of some relevant operators. Our attention will be restricted to the case $N \geqslant 2$, when there is no need to expand Einstein-Hilbert and cosmological terms. The expansions of the latter operators can be easily found elsewhere [9-12]. In the expressions below the symmetrization in the pairs of indices $(\mu, v)$ and $(\rho, \sigma)$ is supposed. We present the expansions valid on maximally symmetric spacetimes, with $\Lambda$ a constant parameter characterizing the background curvature according to the formula $\Lambda=R / 4$.

We give the expression for the relevant part of the bilinear form for the action (2)

$$
\frac{1}{\sqrt{|g|}} \frac{\delta^{2} S_{N}}{\delta h_{\mu \nu} \delta h_{\rho \sigma}}=\sum_{n=0}^{N} S_{(n)}^{\prime \prime} \mu \nu, \rho \sigma,
$$

where for $n \geqslant 1$

$$
\begin{aligned}
& S_{(n)}^{\prime \prime} \mu \nu, \rho \sigma=g^{\mu \rho} g^{\nu \sigma}\left[\frac{2 n-3}{3} \Lambda \omega_{n, C}+\frac{2 n-3}{3} \Lambda \omega_{n, \mathrm{~GB}}\right. \\
& \left.+\frac{1}{2} \omega_{n, C} \square\right] \square^{n+1}+g^{\mu \nu} g^{\rho \sigma} \\
& \times\left[-\frac{4 n-5}{18} \Lambda \omega_{n, C}-\frac{2 n-1}{3} \Lambda \omega_{n, \mathrm{~GB}}\right. \\
& \left.-\frac{2(n-2)}{3} \Lambda \omega_{n, R}-\left(\frac{1}{6} \omega_{n, C}-\omega_{n, R}\right) \square\right] \square^{n+1} \\
& +g^{\mu \nu} \nabla^{\rho} \nabla^{\sigma}\left[\frac{2 n-1}{9} \Lambda \omega_{n, C}+\frac{2 n-1}{3} \Lambda \omega_{n, \mathrm{~GB}}\right. \\
& \left.+\frac{8 n+5}{3} \Lambda \omega_{n, R}+\left(\frac{1}{6} \omega_{n, C}-\omega_{n, R}\right) \square\right] \square^{n} \\
& +g^{\rho \sigma} \nabla^{\mu} \nabla^{v}\left[\frac{2 n-3}{9} \Lambda \omega_{n, C}+\frac{2 n-1}{3} \Lambda \omega_{n, \mathrm{~GB}}\right. \\
& \left.+\frac{2 n-3}{3} \Lambda \omega_{n, R}+\left(\frac{1}{6} \omega_{n, C}-\omega_{n, R}\right) \square\right] \square^{n} \\
& +g^{\mu \rho} \nabla^{\mathcal{v}} \nabla^{\sigma}\left[-\frac{4(n-1)}{3} \Lambda \omega_{n, C}\right. \\
& \left.-\frac{2(2 n-1)}{3} \Lambda \omega_{n, \mathrm{~GB}}-\omega_{n, C} \square\right] \square^{n} \\
& +\nabla^{\mu} \nabla^{\mathcal{v}} \nabla^{\rho} \nabla^{\sigma}\left[\frac{4}{9} n \Lambda \omega_{n, C}-\frac{8}{3} n \Lambda \omega_{n, R}\right. \\
& \left.+\frac{1}{3} \omega_{n, C} \square+\omega_{n, R} \square\right] \square^{n-1}+\mathcal{O}\left(\Lambda^{2}\right) .
\end{aligned}
$$


For the gauge-fixing term described in (14), (15), (16) the expansion looks like

$$
\begin{aligned}
& \frac{1}{\sqrt{|g|}} \frac{\delta^{2} S_{\mathrm{gf}}}{\delta h_{\mu \nu} \delta h_{\rho \sigma}}=\frac{1}{\sqrt{|g|}} \frac{\delta^{2}}{\delta h_{\mu \nu} \delta h_{\rho \sigma}} \int d^{4} x \sqrt{|g|} \chi_{\mu} C^{\mu \nu} \chi_{\nu} \\
& =\frac{2 \beta \gamma}{\alpha} g^{\mu \nu} g^{\rho \sigma}\left(\beta \square-\frac{2}{3}(N+1) \Lambda+\beta N \Lambda\right) \square^{N+1} \\
& +g^{\rho \sigma} \nabla^{\mu} \nabla^{\nu}\left(-\frac{2 \gamma \beta}{\alpha}(\square+N \Lambda)+\frac{4}{3 \alpha}(1+\gamma N) \Lambda\right) \square^{N} \\
& +\frac{2 \gamma \beta}{\alpha} g^{\mu \nu} \nabla^{\rho} \nabla^{\sigma}\left(-\square+\frac{5 N+8}{3} \Lambda\right) \square^{N} \\
& +\frac{2}{\alpha} g^{\mu \rho} \nabla^{v} \nabla^{\sigma}\left(\square-\frac{5 N+8}{3} \Lambda\right) \square^{N} \\
& +\frac{2(\gamma-1)}{\alpha} \nabla^{\mu} \nabla^{\nu} \nabla^{\rho} \nabla^{\sigma}\left(\square-\frac{5}{3} N \Lambda\right) \square^{N-1} \\
& +O\left(\Lambda^{2}\right) .
\end{aligned}
$$

This expression corresponds to arbitrary values of gaugefixing parameters, but for the practical calculations we used (19). Let us stress that the final result is independent on the choice of $\alpha, \beta$ and $\gamma$. The expressions (52) and (53) were obtained from the self-adjoint forms of the variational derivative operators after the commutations of derivatives and expansions in powers of $\Lambda$.

Now we want to perform an additional check. Since from the beginning of our computation, we used "the best" basis in (2), where the contributions to the propagators from particular terms diagonalize, we were not able to do the check of the generalized GB term directly from the combination of terms with Ricci scalar, tensor and Riemann tensor. Instead we had separately terms in the action with Ricci scalars, Weyl tensors, and this generalized GB term. Now we may investigate the effect of the generalized GB term on flat spacetime. Its second variation should vanish (after integration by parts, or equivalently in the self-adjoint form of the second variational derivative). One easily checks that indeed for our variations the contribution from the generalized GB is clearly zero on the flat spacetime. In the formula (52) there are no terms proportional to $\omega_{n, \mathrm{~GB}}$, which would survive on flat spacetime, where $\Lambda \rightarrow 0$. The only terms with $\omega_{n, \mathrm{~GB}}$ are proportional to $\Lambda$. So we proved that in any dimension the contribution to graviton's propagator around flat spacetime from a generalized Gauss-Bonnet term for any $n$ is zero.

In the rest of this Appendix we present the elements of the tensor $H^{\prime}$ from Eq. (25). In all formulas below the symmetrization in the pair of indices $(\kappa, \lambda)$ is assumed. For the calculation of the linear in curvature part of the effective action it is sufficient to consider $V$ and $U$ tensors. According to the notation introduced in formula (31) we can write

$$
\begin{aligned}
& V_{\kappa \lambda}{ }^{\rho \sigma, \alpha \beta \gamma \delta}=\tilde{V}_{(0) \kappa \lambda^{\rho \sigma, \alpha \beta \gamma \delta}+\Lambda \tilde{V}_{(1) \kappa \lambda}}{ }^{\rho \sigma, \alpha \beta \gamma \delta}, \\
& U_{\kappa \lambda}{ }^{\rho \sigma, \alpha \beta \gamma \delta}=\tilde{U}_{(0) \kappa \lambda^{\rho \sigma, \alpha \beta \gamma \delta}+\Lambda \tilde{U}_{(1) \kappa \lambda}}{ }^{\rho \sigma, \alpha \beta \gamma \delta}
\end{aligned}
$$

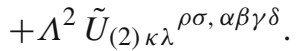

Due to dimensional arguments in the $\tilde{U}_{(0)}$ tensor we would have the appearance of coefficients of the type $\omega_{N-2, i} / \omega_{N, j}$ (where $i, j=R, C$, or GB), while we know from considerations in the main text that this tensor would not contribute to divergences proportional to the linear term in scalar curvature. Similarly, we know that in the $\tilde{U}_{(2)}$ tensor we find coefficients $\omega_{N, i} / \omega_{N, j}$, and again they cannot contribute to the desired divergence because we know from (30) that for divergences we can only take the trace of the $U$ tensor (with no interference terms). Hence we neglect writing the form of these parts of the tensor $U$ and we concentrate below only on the part $\tilde{U}_{(1)}$ that gives contribution.

The list of the corresponding expressions is as follows

$$
\begin{aligned}
& \tilde{V}_{(0) \kappa \lambda} \rho \sigma, \alpha \beta \gamma \delta \\
& =\left[\delta_{\kappa}^{\rho} \delta_{\lambda}^{\sigma} g^{\alpha \beta} g^{\gamma \delta}-\frac{1}{3} g_{\kappa \lambda} g^{\rho \sigma} g^{\alpha \beta} g^{\gamma \delta}\right. \\
& +\frac{1}{3} g^{\rho \sigma} \delta_{\kappa}^{\alpha} \delta_{\lambda}^{\beta} g^{\gamma \delta}+\frac{1}{3} g_{\kappa \lambda} g^{\alpha \rho} g^{\beta \sigma} g^{\gamma \delta} \\
& \left.+\frac{2}{3} \delta_{\kappa}^{\alpha} \delta_{\lambda}^{\beta} g^{\gamma \rho} g^{\delta \sigma}-2 \delta_{\kappa}^{\rho} \delta_{\lambda}^{\alpha} g^{\beta \sigma} g^{\gamma \delta}\right] \frac{\omega_{N-1, C}}{\omega_{N, C}} \\
& +2\left[\delta_{\kappa}^{\alpha} \delta_{\lambda}^{\beta} g^{\gamma \rho} g^{\delta \sigma}-g^{\rho \sigma} \delta_{\kappa}^{\alpha} \delta_{\lambda}^{\beta} g^{\gamma \delta}\right] \frac{\omega_{N-1, R}}{\omega_{N, C}} \\
& +\left[g_{\kappa \lambda} g^{\rho \sigma} g^{\alpha \beta} g^{\gamma \delta}-g_{\kappa \lambda} g^{\alpha \rho} g^{\beta \sigma} g^{\gamma \delta}\right] \frac{\omega_{N-1, R}}{3 \omega_{N, R}}, \\
& \tilde{V}_{(1) \kappa \lambda} \rho \sigma, \alpha \beta \gamma \delta=\frac{2(2 N-3)}{3} \delta_{\kappa}^{\rho} \delta_{\lambda}^{\sigma} g^{\alpha \beta} g^{\gamma \delta} \\
& -\frac{2(3 N-5)}{9} g_{\kappa \lambda} g^{\rho \sigma} g^{\alpha \beta} g^{\gamma \delta}+\frac{3 N+2}{3} g^{\rho \sigma} \delta_{\kappa}^{\alpha} \delta_{\lambda}^{\beta} g^{\gamma \delta} \\
& +2 N \delta_{\kappa}^{\alpha} \delta_{\lambda}^{\beta} g^{\gamma \rho} g^{\delta \sigma}+2\left[(N-1) g^{\rho \sigma} \delta_{\kappa}^{\alpha} \delta_{\lambda}^{\beta} g^{\gamma \delta}\right. \\
& \left.-N \delta_{\kappa}^{\alpha} \delta_{\lambda}^{\beta} g^{\gamma \rho} g^{\delta \sigma}+\frac{1}{3} g_{\kappa \lambda} A^{\alpha \rho, \beta \sigma} g^{\gamma \delta}\right] \frac{\omega_{N, R}}{\omega_{N, C}} \\
& +\frac{2(2 N-1)}{3}\left[\delta_{\kappa}^{\rho} \delta_{\lambda}^{\sigma} g^{\alpha \beta} g^{\gamma \delta}+\frac{1}{3} g_{\kappa \lambda} A^{\alpha \rho, \beta \sigma} g^{\gamma \delta}\right. \\
& \left.+\delta_{\kappa}^{\alpha}\left(g^{\rho \sigma} \delta_{\lambda}^{\beta}-2 \delta_{\lambda}^{\rho} g^{\beta \sigma}\right) g^{\gamma \delta}\right] \frac{\omega_{N, \mathrm{~GB}}}{\omega_{N, C}} \\
& +\frac{2(2 N-1)}{27} g_{\kappa \lambda} A^{\alpha \rho, \beta \sigma} g^{\gamma \delta} \frac{\omega_{N, \mathrm{~GB}}}{\omega_{N, R}} \\
& -\frac{2(9 N+4)}{3} \delta_{\kappa}^{\rho} \delta_{\lambda}^{\alpha} g^{\beta \sigma} g^{\gamma \delta}+\frac{2(6 N+1)}{9} g_{\kappa \lambda} g^{\alpha \rho} g^{\beta \sigma} g^{\gamma \delta},
\end{aligned}
$$

where

$$
A^{\alpha \rho, \beta \sigma}=g^{\alpha \rho} g^{\beta \sigma}-g^{\rho \sigma} g^{\alpha \beta} .
$$


Finally,

$$
\begin{aligned}
& \tilde{U}_{(1) \kappa \lambda}{ }^{\rho \sigma, \alpha \beta \gamma \delta}=2 \delta_{\kappa}^{\rho} \delta_{\lambda}^{\sigma} g^{\alpha \beta} g^{\gamma \delta}\left(\frac{2 N-5}{3} \frac{\omega_{N-1, C}}{\omega_{N, C}}\right. \\
& \left.+\frac{2 N-3}{3} \frac{\omega_{N-1, \mathrm{~GB}}}{\omega_{N, C}}\right) \\
& +g_{\kappa \lambda} g^{\rho \sigma} g^{\alpha \beta} g^{\gamma \delta}\left(-\frac{12 N-31}{27} \frac{\omega_{N-1, C}}{\omega_{N, C}}\right. \\
& \left.-\frac{2(2 N-3)}{9} \frac{\omega_{N-1, \mathrm{~GB}}}{\omega_{N, C}}-\frac{2}{9} \frac{\omega_{N-1, R}}{\omega_{N, C}}\right) \\
& +g_{\kappa \lambda} g^{\rho \sigma} g^{\alpha \beta} g^{\gamma \delta}\left(-\frac{2}{81} \frac{\omega_{N-1, C}}{\omega_{N, R}}\right. \\
& \left.-\frac{2(2 N-3)}{27} \frac{\omega_{N-1, \mathrm{~GB}}}{\omega_{N, R}}-\frac{6 N-19}{27} \frac{\omega_{N-1, R}}{\omega_{N, R}}\right) \\
& +4 \delta_{\kappa}^{\rho} \delta_{\lambda}^{\alpha} g^{\beta \sigma} g^{\gamma \delta}\left(-\frac{2(N-2)}{3} \frac{\omega_{N-1, C}}{\omega_{N, C}}\right. \\
& \left.-\frac{2 N-3}{3} \frac{\omega_{N-1, \mathrm{~GB}}}{\omega_{N, C}}\right) \\
& +2 g_{\kappa \lambda} g^{\alpha \rho} g^{\beta \sigma} g^{\gamma \delta}\left(\frac{6 N-17}{27} \frac{\omega_{N-1, C}}{\omega_{N, C}}\right. \\
& \left.+\frac{2 N-3}{9} \frac{\omega_{N-1, \mathrm{~GB}}}{\omega_{N, C}}-\frac{5}{9} \frac{\omega_{N-1, R}}{\omega_{N, C}}\right) \\
& +2 g_{\kappa \lambda} g^{\alpha \rho} g^{\beta \sigma} g^{\gamma \delta}\left(\frac{4}{81} \frac{\omega_{N-1, C}}{\omega_{N, R}}\right. \\
& \left.+\frac{2 N-3}{27} \frac{\omega_{N-1, \mathrm{~GB}}}{\omega_{N, R}}+\frac{2(6 N-1)}{27} \frac{\omega_{N-1, R}}{\omega_{N, R}}\right) \\
& +2 g^{\rho \sigma} \delta_{\kappa}^{\alpha} \delta_{\lambda}^{\beta} g^{\gamma \delta}\left(\frac{2 N-5}{9} \frac{\omega_{N-1, C}}{\omega_{N, C}}\right. \\
& \left.+\frac{2 N-3}{3} \frac{\omega_{N-1, \mathrm{~GB}}}{\omega_{N, C}}+\frac{2 N-5}{3} \frac{\omega_{N-1, R}}{\omega_{N, C}}\right) \\
& +8 \delta_{\kappa}^{\alpha} \delta_{\lambda}^{\beta} g^{\gamma \rho} g^{\delta \sigma}\left(\frac{N-1}{9} \frac{\omega_{N-1, C}}{\omega_{N, C}}-\frac{2(N-1)}{3} \frac{\omega_{N-1, R}}{\omega_{N, C}}\right),
\end{aligned}
$$

completing the list of necessary formulas.

\section{Appendix B}

We here remind the standard definitions from the review paper [9-11].

The divergent contribution to the quantum effective action is given by:

$$
\begin{aligned}
\Gamma_{\text {div }} & =\frac{i}{2} \operatorname{Det} H^{\prime}=\frac{i}{2} \operatorname{Tr} \ln H^{\prime} \\
& =\frac{i}{2}\left(i \ln L^{2}\right) \int d^{4} x \sqrt{|g|}\left(\beta_{C} C^{2}+\beta_{R} R^{2}+\beta_{G} R+\beta_{\Lambda_{\mathrm{cc}}}\right)
\end{aligned}
$$

$$
\begin{aligned}
= & -\frac{1}{2} \ln L^{2} \int d^{4} x \sqrt{|g|} \\
& \times\left(\beta_{C} C^{2}+\beta_{R} R^{2}+\beta_{G} R+\beta_{\Lambda_{\mathrm{cc}}}\right),
\end{aligned}
$$

where

$$
\begin{aligned}
\ln L^{2} & =\frac{1}{2-\omega}, \quad \omega=2-0^{+}=2-\varepsilon=\frac{n}{2} \\
& \Longrightarrow \ln L^{2}=\frac{1}{\varepsilon}>0,
\end{aligned}
$$

where $L=\Lambda / \mu, \Lambda$ is the cut-off scale and $\mu$ the renormalization scale.

Moreover,

$$
\begin{aligned}
n & =2 \omega=2\left(2-0^{+}\right)=2(2-\varepsilon)=4-2 \varepsilon \\
& \Longrightarrow \ln L^{2}=\frac{2}{4-n}=\frac{1}{\varepsilon},
\end{aligned}
$$

where $n$ is the dimensionality of spacetime. Then Eq. (59) turns into

$$
\Gamma_{\mathrm{div}}=-\frac{1}{2 \varepsilon} \int d^{4} x \sqrt{|g|}\left(\beta_{C} C^{2}+\beta_{R} R^{2}+\beta_{G} R+\beta_{\Lambda_{\mathrm{cc}}}\right) .
$$

Finally, we give here the divergent contributions to the trace of the logarithm of $H^{\prime}$ operator for the following different values of $N$ in dimensional regularization scheme.

For $N \geqslant 2$ :

$$
\begin{aligned}
\left.\operatorname{Tr} \ln H^{\prime}\right|_{\mathrm{div}}= & \frac{i \ln L^{2}}{16 \pi^{2}} \int d^{4} x \sqrt{|g|}(-1)\left\{\frac{5 \omega_{N-1, C}}{6 \omega_{N, C}}+\frac{\omega_{N-1, R}}{6 \omega_{N, R}}\right. \\
& +(2 N-1)\left[\frac{5 \omega_{N-1, C}}{6 \omega_{N, C}^{2}}-\frac{\omega_{N-1, R}}{18 \omega_{N, R}^{2}}\right] \omega_{N, \mathrm{~GB}} \\
& \left.+(2 N-3)\left[\frac{5}{6 \omega_{N, C}}-\frac{1}{18 \omega_{N, R}}\right] \omega_{N-1, \mathrm{~GB}}\right\} R \\
& +O\left(\mathcal{R}^{0}, \mathcal{R}^{2}\right) .
\end{aligned}
$$

For $N=1$ :

$$
\begin{aligned}
\left.\operatorname{Tr} \ln H^{\prime}\right|_{\text {div }}= & \frac{-i \ln L^{2}}{16 \pi^{2}} \int d^{4} x \sqrt{|g|}\left\{\frac{5 \omega_{0, C}}{6 \omega_{1, C}}+\frac{\omega_{0, R}}{2 \omega_{1, R}}\right. \\
& \left.-\frac{5 \omega_{0, R}}{\omega_{1, C}}+\left(\frac{5 \omega_{0, C}}{6 \omega_{1, C}^{2}}-\frac{\omega_{0, R}}{18 \omega_{1, R}^{2}}\right) \omega_{1, \mathrm{~GB}}\right\} R \\
& +O\left(\mathcal{R}^{0}, \mathcal{R}^{2}\right) .
\end{aligned}
$$

For $N=0$ :

$$
\begin{aligned}
\left.\operatorname{Tr} \ln H^{\prime}\right|_{\text {div }}= & \frac{i \ln L^{2}}{16 \pi^{2}} \int d^{4} x \sqrt{|g|}\left(-\frac{5 \omega_{\mathrm{EH}} \omega_{R}}{2 \omega_{C}^{2}}-\frac{13 \omega_{\mathrm{EH}}}{12 \omega_{C}}\right. \\
& \left.+\frac{\omega_{\mathrm{EH}}}{12 \omega_{R}}\right) R+O\left(\mathcal{R}^{0}, \mathcal{R}^{2}\right)
\end{aligned}
$$


And for $N \geqslant 2$ (divergence of the cosmological constant term):

$$
\begin{aligned}
\left.\operatorname{Tr} \ln H^{\prime}\right|_{\operatorname{div}}= & \frac{i \ln L^{2}}{16 \pi^{2}} \int d^{4} x \sqrt{|g|}\left(\frac{5 \omega_{N-2, C}}{\omega_{N, C}}+\frac{\omega_{N-2, R}}{\omega_{N, R}}\right. \\
& \left.-\frac{5 \omega_{N-1, C}^{2}}{2 \omega_{N, C}^{2}}-\frac{\omega_{N-1, R}^{2}}{2 \omega_{N, R}^{2}}\right)+O\left(\mathcal{R}^{1}\right),
\end{aligned}
$$

where by $\mathcal{R}$ above we mean a general gravitational curvature tensor (Ricci scalar, tensor or Riemann tensor).

\section{Appendix C}

Let us comment on the interesting issue of stability in the theory with higher derivatives and complex poles.

The problem of stability of classical solutions is the most difficult one for the theories with higher derivatives. There are different approaches to this important problem. Let us describe the possibility which takes place within the LeeWick construction [22,23]. Indeed, as far as one accepts this approach, the problem is greatly alleviated. The Lee-Wick proposal [22,23] consists of removing the instabilities by requiring, as a boundary condition, that the growing modes do not appear on-shell (see also the discussion below about tree-level and perturbative unitarity). As a consequence of eliminating the exponentially growing states, a causal effects occur, just as in the classical theory of electron, when runaway modes have to be eliminated. This is not surprising: a future boundary condition has been imposed and the initial state must be such as to eliminate any growing modes that would otherwise be created. This topic has been extensively discussed in the literature; the results may look nonconventional but do not appear to conflict with any experimental observations, nor do any paradoxes or inconsistencies arise.

Furthermore, the linear stability analysis comes together with the unitarity issue because both have to do with the $S$ matrix at perturbative level. The former at tree-level while the latter at any order in the loop expansion. The problem is to define a unitary $S$-matrix, but fortunately, this was done by Lee and Wick. Indeed, the evolution operator $U\left(t, t_{0}\right)$ is not unitary and, moreover, it becomes unbounded as $t \rightarrow \pm \infty$. However, the physical states are defined so as to exclude the complex-mass particles from the asymptotic states and contain only real-mass particles. The $S$-matrix is obtained by subtracting from $U$ the terms that grow exponentially, and is shown to be unitary [22,23].

Equivalently, the $S$-matrix is obviously unitary in the whole Hilbert space of real and complex states, then, in order to prove the unitarity of the $S$-matrix in the physical subspace of real states, we have only to prove that if the initial state is physical, then the final state is physical too. The key point here is that the energy conservation comes together with the $S$-matrix, and, therefore, if the initial state has real energy also the final state has real energy, otherwise the energy would get an imaginary part. The orthogonality between real and complex energy states is satisfied on both sides of the unitarity equation $T-T^{\dagger}=i T^{\dagger} T$, which comes from $S^{\dagger} S=1$ and $S=1+i T$. It is easy to see that the $S$-matrix is unitary in the physical subspace of real states (in the minimal Lee-Wick gravitational theory we only have the graviton state). The complex-mass particles appear only in virtual states which never go on-shell and never become asymptotic states. We can have complex conjugate ghost pairs with a positive norm and real energy in a special Lorentz frame, but their energy is complex in general Lorentz frames. These states have to be projected out as well but the argument above does not change because such states are in the Hilbert space of complex ghosts. Indeed, they are tensor products of complex states. Finally, we can get negative norm states combining complex ghosts' states but again these states are complex and, therefore, outside the physical subspace.

The classical stability is the most trivial application of the unitarity analysis namely, we use the tree-level $S$-matrix scattering amplitude. Of course, the action is not sufficient to define the theory and we have to add the Lee-Wick prescription to get something meaningful. Naively, there are runaway solutions, but when the Hilbert space of the asymptotic states is properly projected there are no such instabilities. A much more difficult task is to prove the perturbative unitarity. However, the latter was proved by Cutkosky, Landshoff, Olive, and Polkinghorne (CLOP) in Ref. [48] as it is shortly summarized in the paper [46] of our group. Finally, the CLOP prescription allows for the Wick rotation (see also [49]), which justifies our computation also in the Minkowski space. Other forms of "non-analytic" continuation from Minkowskian to Euclidean signature can be also found in the literature. These results make the theory published in [46] a very good proposal for quantum gravity $[46,47]$.

At classical level, another well-defined prescription, similar to the Lee-Wick one, to remove the runaway solutions was proposed in Ref. [90].

It is impossible to say that the solutions of the stability problem in higher derivative models is ultimately achieved by the considerations presented above. However, at the presentday level of understanding these considerations look useful and may be instructive in constructing a consistent theory of quantum gravity. Another approach to the problem of stability has been recently discussed in [91-94].

\section{Appendix D}

As we have explained in the main part of the paper, the beta functions for the cases $N \geqslant 1$ are universal in the sense 
they are independent on the choice of gauge-fixing and/or parametrization of a quantum field. This feature is in a sharp contrast with the situation in quantum gravity based on Einstein's general relativity (see recent discussion in [95] and further references therein) and four-derivative quantum gravity.

One can naturally ask whether some other type of ambiguity can be detected in the super-renormalizable model of quantum gravity. Let us consider, for instance, the transformation of the metric

$g_{\mu \nu} \longrightarrow g_{\mu \nu}^{\prime}=g_{\mu \nu}+\mathcal{X}_{\mu \nu}$.

The question is whether such a transformation can modify the beta functions. ${ }^{3}$ The transformation of this sort has been discussed long time ago in relation to renormalization of quantum general relativity [4] (see also more general discussion in [96]), but it is useful to analyze it in the essentially more general context.

It is evident that one can apply (67) in two different ways. The first one is not to assume any relation between $\mathcal{X}_{\mu \nu}$ and the quantum corrections. In this case, it is clear that choosing the trace of the tensor $\mathcal{X}_{\mu \nu}$ in a special way one can modify all terms in the action, except the cosmological constant term (in a case we do not like to modify it). In order to understand this, let us present the total Lagrangian of the theory (both the gravitational and matter parts) in the form

$L_{\text {tot }}=\omega_{\mathrm{cc}}+L_{A}$.

The total classical action is

$S_{\text {tot }}=\int d^{4} x \sqrt{|g|} L_{\text {tot }}$.

Under the replacement (67) the determinant in (69) transforms according to the Liouville's formula

$g^{\prime}=g \times \exp \left\{\mathcal{X}-\frac{1}{2} \mathcal{X}_{\mu \nu}^{2}+\frac{1}{3} \mathcal{X}_{\mu \nu}^{3}-\frac{1}{4} \mathcal{X}_{\mu \nu}^{4}+\cdots\right\}$,

where the contractions are done with the metric, e.g., $\mathcal{X}=$ $g^{\mu \nu} \mathcal{X}_{\mu \nu}, \mathcal{X}_{\mu \nu}^{2}=g^{\mu \alpha} g^{\nu \beta} \mathcal{X}_{\mu \nu} \mathcal{X}_{\alpha \beta}$, etc. According to the last formulas, the choice

$\mathcal{X}_{\mu \nu}=-\frac{L_{A}}{2 \omega_{\mathrm{cc}}} g_{\mu \nu}$

eliminates all the terms in $S_{\text {tot }}\left(g^{\prime}\right)$, which are linear in $L_{A}$, these terms being gravitational or matter Lagrangians.

After the operation (67), in terms of the metric $g_{\mu \nu}$ the theory changes dramatically. At first order in $L_{A}$ all the terms in the action disappear and there is only the cosmological constant remaining. Then the next order terms $O\left(L_{A}^{2}\right)$ can be eliminated by further tuning of the tensor $\mathcal{X}_{\mu \nu}$ which becomes an infinite series in $L_{A}$. The proof that it is possible to do

\footnotetext{
3 We acknowledge the stimulating requirement of one of the anonymous referees to include the discussion on this issue.
}

this non-perturbatively is left to the interested reader as an exercise.

At this level, the operation (67) has nothing to do with quantum corrections, it is a purely classical change of the metric variable which kills all terms in the action except the $\omega_{\mathrm{cc}}$, namely the cosmological constant term. One can, of course, make changes in the operation (67), e.g., kill only purely gravitational terms, or purely matter terms, or make another selection. In this case there will be obvious complications, but let us skip this part for the sake of brevity.

The application of the operation (67) at the quantum level opens new possibilities for further speculations. In this case the classical action (69) is replaced by the quantum ana$\log$ called effective action (see [61] for the details in curved spacetime and in quantum gravity),

$\Gamma=S_{\mathrm{tot}}+\bar{\Gamma}, \quad \bar{\Gamma}=\sum_{k=1}^{\infty} \hbar^{k} \bar{\Gamma}^{(k)}$,

where the last sum corresponds to the loop expansion. One can link the operation (67) with the parameter of the loop expansion $\hbar$. It is clear that taking $\mathcal{X}_{\mu \nu}=O(\hbar)$ one can, for example, kill completely or partially, or just modify the one-loop corrections, while the classical terms $L_{A}$ will not be affected at all. Furthermore, taking $\mathcal{X}_{\mu \nu}$ to be an infinite series in $\hbar$, one can arbitrarily (or almost so) modify higher loop corrections too. One can perform a transformation (67) with the loop parameter $\hbar$ and then all the beta functions will be affected (can be canceled, for instance), but this would be as artificial as in the general classical case considered above.

One can make speculations on the basis of the operation (67). For instance, in quantum gravity one can start with the action with four derivatives, or with the super-renormalizable action (2), calculate quantum corrections and then postulate that the physical observable metric is not the original one $g_{\mu \nu}$, but some other one, obtained by the operation (67), such that the resulting theory has no ghosts. One can see an example of this procedure described in the recent paper Ref. [97]. This approach to quantum gravity has only three problems: (i) the procedure of changing the field in an arbitrary way is not a change of variables (classical or quantum) and does not belong to the conventional quantum field theory; (ii) in some cases it requires infinitely precise fine-tuning of infinitely many parameters, as it was recently discussed in [51] in relation to string theory and Zwiebach transformation [67-69]; (iii) there are serious ambiguities in the resulting action since there are many higher derivative terms which do not produce ghosts, and which can be eliminated or not, depending on our will [98].

As far as our interest in the present paper is related to the one-loop beta functions for the Newton constant $G$ and the cosmological constant $\Lambda_{\mathrm{cc}}$, we can restrict our attention 
to the corresponding terms only. ${ }^{4}$ Since we will deal with the $\overline{\mathrm{MS}}$-scheme of renormalization, we can also restrict our attention to the corresponding version of the renormalization group in curved space, as described in a pedagogical form in [61]. In order to simplify things as much as possible, consider the theory (2) with $N \geqslant 3$, such that one-loop beta functions are exact and non-zero only for the parameters $\omega_{i}=\left(\omega_{\mathrm{EH}}, \omega_{\mathrm{cc}}, \omega_{0, R}, \omega_{0, C}, \omega_{0, \mathrm{~GB}}\right)$.

Then the simplest procedure is to take the quantum effective action as the renormalization group improved classical action

$\Gamma \approx S_{\mathrm{tot}}^{\mathrm{RG}}=S_{\mathrm{tot}}\left(\omega_{i}(t)\right)$,

where in the one-loop approximation the corresponding expressions are

$$
\begin{aligned}
\omega_{1}(t) & =\omega_{\mathrm{EH}}(t)=\omega_{\mathrm{EH}}(0)+\beta_{G} t, \\
\omega_{2}(t) & =\omega_{\mathrm{cc}}(t)=\omega_{\mathrm{cc}}(0)+\beta_{\mathrm{cc}} t, \\
\omega_{3,4,5}(t) & =\omega_{3,4,5}(0)+\beta_{3,4,5} t .
\end{aligned}
$$

Actually, the last three beta functions were not calculated yet, but we do not need them now. In these formulas the "RG time" $t$ can be understood in two different ways. Taking the renormalization group in terms of the renormalization parameter $\mu$, we can define $t=\ln \left(\mu / \mu_{0}\right)$, such that the value $t=0$ corresponds to the reference scale $\mu_{0}$. On the other hand, it is always necessary to relate an artificial parameter $\mu$ to some physical quantity. In curved space the unique well-developed identification is to associate $t$ with the parameter of the global rescaling of the metric [30] (see also [31,61] for a consistent treatment and full details),

$g_{\mu \nu} \longrightarrow g_{\mu \nu} e^{2 t}$.

Let us use this fact as a hint to explore how the operation (67) can affect the beta functions $\beta_{G}$ and $\beta_{\mathrm{cc}}$.

As far as the beta functions are related to quantum corrections, we can regard both $\beta_{G}$ and $\beta_{\mathrm{cc}}$ as $O(\hbar)$. Then the first two relations in (74) can be recast as

$$
\begin{aligned}
\omega_{\mathrm{EH}}(t) & =\omega_{\mathrm{EH}}(0) \exp \left\{\frac{\beta_{G} t}{\omega_{\mathrm{EH}}(0)}\right\}, \\
\omega_{\mathrm{cc}}(t) & =\omega_{\mathrm{cc}}(0) \exp \left\{\frac{\beta_{\mathrm{cc}} t}{\omega_{\mathrm{cc}}(0)}\right\} .
\end{aligned}
$$

Then the renormalization-group improved classical action (73) becomes

$S_{\mathrm{tot}}^{\mathrm{RG}}=\int d^{4} x \sqrt{|g|}\left\{\omega_{\mathrm{cc}}(t)+\omega_{\mathrm{EH}}(t) R+O\left(\mathcal{R}^{2}\right)\right\}$.

\footnotetext{
4 The interested reader can easily make generalizations to other terms in the gravitational or matter fields actions and explore the ambiguity of the corresponding beta functions.
}

Consider a very particular version of (67),

$g_{\mu \nu} \longrightarrow g_{\mu \nu}^{\prime}=e^{2 \alpha t} g_{\mu \nu}$,

where $\alpha$ is an arbitrary parameter of the first order in $\hbar$. Replacing (78) into (77), after a small algebra we find that the beta functions get modified according to

$\beta_{\mathrm{cc}} \longrightarrow \beta_{\mathrm{cc}}^{\prime}=\beta_{\mathrm{cc}}+4 \alpha \omega_{\mathrm{cc}}(0)$,

$\beta_{G} \longrightarrow \beta_{G}^{\prime}=\beta_{G}+2 \alpha \omega_{\mathrm{EH}}(0)$.

Since we are interested only in the first order in $\hbar$, it is possible to trade $\omega_{\mathrm{cc}}(0)$ and $\omega_{\mathrm{EH}}(0)$ in expressions (79) to fully $t$ dependent $\omega_{\mathrm{cc}}(t)$ and $\omega_{\mathrm{EH}}(t)$. And then it is clear that the quantity which has $\alpha$-independent derivative with respect to $t$ is the dimensionless ratio

$\frac{\omega_{\mathrm{cc}}(t)}{\omega_{\mathrm{EH}}^{2}(t)} \sim G \Lambda_{\mathrm{cc}}$.

By coincidence, this is the same combination that does not depend on the choice of gauge-fixing in four-derivative gravity and appears in the on-shell renormalization group in perturbative quantum Einstein's general relativity [8] (see also [9-11,39] for further details in the first case and [95] in the last one).

Does it mean that the ambiguity related to (78) and the one related to the choice of the gauge-fixing or parametrization of quantum fields are somehow related? In our opinion the answer is negative. As we have argued above, the transformation (67) can produce much more significant changes in the theory at both classical and quantum level than the change of the gauge-fixing. For instance, it is an easy exercise to show that (67) can change the sign of the beta function for the running charges in QED or in QCD. The corresponding beta functions do not depend on the gauge-fixing and do not have any relation to gravity or metric, so this operation is obviously senseless. In general, the operation (67) and its particular version (78) are not related to quantum field theory. These operations with the background quantities are not related to legitimate changes of quantum variables in the path integral formalism. The reason is that the corresponding transformation should be done for the background metric and is not the change of quantum variables. One can say that the transformations (67) should be "forbidden" if we intend to explore the quantum field theory formalism. All in all, (67) is a technical trick which is not related to quantization at all.

\section{References}

1. G. t'Hooft, M. Veltman, One loop divergencies in the theory of gravitation. Ann. Inst. H. Poincare A 20, 69 (1974)

2. S. Deser, P. van Nieuwenhuisen, One loop divergences of quantized Einstein-Maxwell fields. Phys. Rev. D 10, 401 (1974)

3. S. Deser, P. van Nieuwenhuisen, Nonrenormalizability of the quantized Dirac-Einstein system. Phys. Rev. D 10, 411 (1974) 
4. R.E. Kallosh, O.V. Tarasov, I.V. Tyutin, One loop finiteness of quantum gravity off mass shell. Nucl. Phys. B 137, 145 (1978)

5. M.H. Goroff, A. Sagnotti, The ultraviolet behavior of Einstein gravity. Nucl. Phys. B 266, 709 (1986)

6. A.E.M. van de Ven, Two loop quantum gravity. Nucl. Phys. B 378, 309 (1992)

7. J. Julve, M. Tonin, Quantum gravity with higher derivative terms. Nuovo Cim. B 46, 137 (1978)

8. E.S. Fradkin, A.A. Tseytlin, Renormalizable asymptotically free quantum theory of gravity. Nucl. Phys. B 201, 469 (1982)

9. I.G. Avramidi, A.O. Barvinsky, Asymptotic freedom in higher derivative quantum gravity. Phys. Lett. B 159, 269 (1985)

10. I.G. Avramidi, Asymptotic behavior of the quantum theory of gravity with higher order derivatives (In Russian). Sov. J. Nucl. Phys. 44, 255 (1986)

11. I.G. Avramidi, Covariant methods for the calculation of the effective action in quantum field theory and investigation of higherderivative quantum gravity. Ph.D. thesis, Moscow University, 1986. arXiv:hep-th/9510140

12. G. de Berredo-Peixoto, I.L. Shapiro, Higher derivative quantum gravity with Gauss-Bonnet term. Phys. Rev. D 71, 064005 (2005)

13. D.M. Capper, D. Kimber, An ambiguity in one loop quantum gravity. J. Phys. A 13, 3671 (1980)

14. I. Antoniadis, P.O. Mazur, E. Mottola, Conformal symmetry and central charges in four-dimensions. Nucl. Phys. B 388, 627 (1992)

15. G. de Berredo-Peixoto, I.L. Shapiro, Conformal quantum gravity with the Gauss-Bonnet term. Phys. Rev. D 70, 044024 (2004)

16. K.S. Stelle, Renormalization of higher derivative quantum gravity. Phys. Rev. D 16, 953 (1977)

17. E. Tomboulis, $1 / \mathrm{N}$ expansion and renormalization in quantum gravity. Phys. Lett. B 70, 361 (1977)

18. E. Tomboulis, Renormalizability and asymptotic freedom in quantum gravity. Phys. Lett. B 97, 77 (1980)

19. E. Tomboulis, Unitarity in higher derivative quantum gravity. Phys. Rev. Lett. 52, 1173 (1984)

20. A. Salam, J. Strathdee, Remarks on high-energy stability and renormalizability of gravity theory. Phys. Rev. D 18, 4480 (1978)

21. I. Antoniadis, E.T. Tomboulis, Gauge invariance and unitarity in higher derivative quantum gravity. Phys. Rev. D 33, 2756 (1986)

22. T.D. Lee, G.C. Wick, Finite theory of quantum electrodynamics. Phys. Rev. D 2, 1033 (1970)

23. T.D. Lee, G.C. Wick, Negative metric and the unitarity of the $\mathrm{S}$ matrix. Nucl. Phys. B 9, 209 (1969)

24. D.A. Johnston, Sedentary ghost poles in higher derivative gravity. Nucl. Phys. B 297, 721 (1988)

25. A. Codello, R. Percacci, Fixed points of higher derivative gravity. Phys. Rev. Lett. 97, 221301 (2006). arXiv:hep-th/0607128

26. A. Codello, R. Percacci, L. Rachwal, A. Tonero, Computing the effective action with the functional renormalization group. Eur. Phys. J. C 76(4), 226 (2016). arXiv:hep-th/1505.03119

27. P.M. Lavrov, I.L. Shapiro, On the functional renormalization group approach for Yang-Mills fields. JHEP 1306, 086 (2013). arXiv:hep-th/1212.2577

28. I.L. Shapiro, J. Solà, On the possible running of the cosmological 'constant'. Phys. Lett. B 682, 105 (2009). arXiv:hep-th/0910.4925

29. D.C. Rodrigues, P.S. Letelier, I.L. Shapiro, Galaxy rotation curves from general relativity with renormalization group corrections. JCAP 1004, 020 (2010). arXiv:0911.4967 [astro-ph.CO]

30. B.L. Nelson, P. Panangaden, Scaling behavior of interacting quantum fields in curved space-time. Phys. Rev. D 25, 1019 (1982)

31. I.L. Buchbinder, Renormalization group equations in curved spacetime. Theor. Math. Phys. 61, 393 (1984)

32. J. Donoghue, Leading quantum correction to the Newtonian potential. Phys. Rev. Lett. 72, 2996 (1994)

33. J. Donoghue, General relativity as an effective field theory: the leading quantum corrections. Phys. Rev. D 50, 3874 (1994)
34. Y. Iwasaki, Quantum theory of gravitation vs. classical theory: fourth-order potential. Prog. Theor. Phys. 46, 1587 (1971)

35. I.L. Shapiro, Asymptotically finite theories and the screening of cosmological constant by quantum effects. Phys. Lett. B 329, 181 (1994)

36. R. Jackiw, C. Nunez, S.-Y. Pi, Quantum relaxation of the cosmological constant. Phys. Lett. A 347, 47 (2005)

37. M. Niedermaier, M. Reuter, The asymptotic safety scenario in quantum gravity. Living Rev. Relativ. 9, 5 (2006)

38. C. Farina, W.J.M. Kort-Kamp, S. Mauro, I.L. Shapiro, Dynamics of the Laplace-Runge-Lenz vector in the quantum-corrected Newton gravity. Phys. Rev. D 83, 124037 (2011). arXiv:1101.5611 [gr-qc]

39. I.L. Shapiro, A.G. Zheksenaev, Gauge dependence in higher derivative quantum gravity and the conformal anomaly problem. Phys. Lett. B 324, 286 (1994)

40. N. Ohta, R. Percacci, A.D. Pereira, Gauges and functional measures in quantum gravity I: Einstein theory. JHEP 1606, 115 (2016). arXiv:1605.00454 [hep-th]

41. N. Ohta, R. Percacci, A.D. Pereira, Gauges and functional measures in quantum gravity II: higher derivative gravity. Eur. Phys. J. C 77(9) 611 (2017). arXiv:hep-th/1610.07991

42. N. Ohta, R. Percacci, Higher derivative gravity and asymptotic safety in diverse dimensions. Class. Quantum Gravity 31, 015024 (2014). arXiv:1308.3398 [hep-th]

43. M. Asorey, J.L. López, I.L. Shapiro, Some remarks on high derivative quantum gravity. Int. J. Mod. Phys. A 12, 5711 (1997)

44. N.V. Krasnikov, Nonlocal Gauge theories. Theor. Math. Phys. 73, 1184 (1987)

45. Y.V. Kuz'min, The Convergent Nonlocal Gravitation (in Russian). Sov. J. Nucl. Phys. 50, 1011 (1989). [Yad. Fiz. 50, 1630 (1989)]

46. L. Modesto, I.L. Shapiro, Superrenormalizable quantum gravity with complex ghosts. Phys. Lett. B 755, 279 (2016). arXiv:1512.07600 [hep-th]

47. L. Modesto, Super-renormalizable or finite Lee-Wick quantum gravity. Nucl. Phys. B 909, 584 (2016). arXiv:1602.02421 [hepth]

48. R.E. Cutkosky, P.V. Landshoff, D.I. Olive, J.C. Polkinghorne, A non-analytic S matrix. Nucl. Phys. B 12, 281 (1969)

49. B. Grinstein, D. O'Connell, M.B. Wise, Causality as an emergent macroscopic phenomenon: the Lee-Wick O(N) model. Phys. Rev. D 79, 105019 (2009). arXiv:0805.2156 [hep-th]

50. L. Modesto, T. de Paula Netto, I.L. Shapiro, On Newtonian singularities in higher derivative gravity models. JHEP 1504, 098 (2015). arXiv:1412.0740 [hep-th]

51. A. Accioly, B.L. Giacchini, I.L. Shapiro, Low-energy effects in a higher-derivative gravity model with real and complex massive poles. Phys. Rev. D 96, 104004 (2017). arXiv:1610.05260 [gr-qc]

52. B.L. Giacchini, On the cancellation of Newtonian singularities in higher-derivative gravity. Phys. Lett. B 766, 306 (2017). arXiv: 1609.05432 [hep-th]

53. A. Accioly, B.L. Giacchini, I.L. Shapiro, On the gravitational seesaw in higher-derivative gravity. Eur. Phys. J. C 77(8), 540 (2017). arXiv: 1604.07348 [gr-qc]

54. L. Modesto, Super-renormalizable quantum gravity. Phys. Rev. D 86, 044005 (2012). arXiv:1107.2403 [hep-th]

55. L. Modesto, Super-renormalizable multidimensional quantum gravity. Astron. Rev. 8(2), 4 (2013). arXiv:1202.3151 [hep-th]

56. L. Modesto, Super-renormalizable higher-derivative quantum gravity. arXiv:1202.0008 [hep-th]

57. L. Modesto, L. Rachwal, Super-renormalizable and finite gravitational theories. Nucl. Phys. B 889, 228 (2014). arXiv:1407.8036 [hep-th]

58. L. Modesto, L. Rachwal, Universally finite gravitational and gauge theories. Nucl. Phys. B 900, 147 (2015). arXiv:1503.00261 [hepth] 
59. I.L. Shapiro, Counting ghosts in the "ghost-free" nonlocal gravity. Phys. Lett. B 744, 67 (2015). arXiv:1502.00106 [hep-th]

60. B.L. Voronov, P.M. Lavrov, I.V. Tyutin, Canonical transformations and the gauge dependence in general gauge theories (in Russian). Sov. J. Nucl. Phys. 36, 498 (1982)

61. I.L. Buchbinder, S.D. Odintsov, I.L. Shapiro, Effective action in quantum gravity (IOP Publishing, Bristol, 1992)

62. A.O. Barvinsky, G.A. Vilkovisky, The generalized SchwingerDeWitt technique in gauge theories and quantum gravity. Phys. Rep. 119, 1 (1985)

63. S. Ichinose, Renormalization of Polyakov's two-dimensional quantum gravity. Phys. Lett. B 251, 49 (1990)

64. A.O. Barvinsky, Nonlocal action for long distance modifications of gravity theory. Phys. Lett. B 572, 109 (2003). arXiv:hep-th/0304229

65. M. Eran, Higher-derivative Gauge And Gravitational Theories (supersymmetry). Ph.D. thesis, University of California, Los Angeles, 1998

66. A.A. Tseytlin, On singularities of spherically symmetric backgrounds in string theory. Phys. Lett. B 363, 223 (1995). arXiv:hep-th/9509050

67. B. Zwiebach, Curvature squared terms and string theories. Phys. Lett. B 156, 315 (1985)

68. S. Deser, A.N. Redlich, String induced gravity and ghost freedom. Phys. Lett. B 176, 350 (1986)

69. A.A. Tseytlin, Ambiguity in the effective action in string theories. Phys. Lett. B 176, 92 (1986)

70. E.T. Tomboulis, Superrenormalizable gauge and gravitational theories. arXiv:hep-th/9702146

71. G.V. Efimov, Nonlocal interactions of quantum fields (In Russian) (Nauka, Moscow, 1977)

72. P. Dona, S. Giaccari, L. Modesto, L. Rachwal, Y. Zhu, Scattering amplitudes in super-renormalizable gravity. JHEP 08, 038 (2015). arXiv:1506.04589 [hep-th]

73. L. Modesto, M. Piva, L. Rachwal, Finite quantum gauge theories. Phys. Rev. D 94(2), 025021 (2016). arXiv:1506.06227 [hep-th]

74. L. Modesto, L. Rachwal, Exact solutions and spacetime singularities in nonlocal gravity. JHEP 1512, 173 (2015). arXiv:1506.08619 [hep-th]

75. S. Giaccari, L. Modesto, L. Rachwal, Y. Zhu, Finite entanglement entropy of black holes. Eur. Phys. J. C 78(6), 459 (2018). arXiv:1512.06206 [hep-th]

76. A.S. Koshelev, L. Modesto, L. Rachwal, A.A. Starobinsky, Occurrence of exact $R^{2}$ inflation in non-local UV-complete gravity. JHEP 1611, 067 (2016). arXiv:1604.03127 [hep-th]

77. L. Modesto, L. Rachwal, Finite conformal quantum gravity and nonsingular spacetimes. arXiv: 1605.04173 [hep-th]

78. C. Bambi, L. Modesto, L. Rachwal, Spacetime completeness of non-singular black holes in conformal gravity. JCAP 1705(05), 003 (2017). arXiv:1611.00865 [gr-qc]

79. C. Bambi, L. Modesto, S. Porey, L. Rachwal, Black hole evaporation in conformal gravity. JCAP 1709(09), 033 (2017). arXiv: $1611.05582 \mathrm{v} 1$ [gr-qc]

80. C. Bambi, L. Modesto, S. Porey, L. Rachwal, Formation and evaporation of an electrically charged black hole in conformal gravity. Eur. Phys. J. C 78(2), 116 (2018). arXiv:1711.04575 [gr-qc]
81. G. Calcagni, M. Montobbio, G. Nardelli, Localization of nonlocal theories. Phys. Lett. B 662, 285 (2008). arXiv:0712.2237 [hep-th]

82. G. Calcagni, G. Nardelli, Non-local gravity and the diffusion equation. Phys. Rev. D 82, 123518 (2010). arXiv:1004.5144 [hep-th]

83. G. Calcagni, L. Modesto, Nonlocal quantum gravity and M-theory. Phys. Rev. D 91(12), 124059 (2015). arXiv:1404.2137 [hep-th]

84. T. Biswas, E. Gerwick, T. Koivisto, A. Mazumdor, Towards singularity and ghost free theories of gravity. Phys. Rev. Lett. 108, 031101 (2012). arXiv: 1110.5249 [gr-qc]

85. T. Biswas, A. Conroy, A.S. Koshelev, A. Mazumdor, Generalized ghost-free quadratic curvature gravity. Class. Quantum Gravity 31, 015022 (2014). Erratum: Class. Quantum Gravity 31, 159501 (2014). arXiv:1308.2319 [hep-th]

86. I.L. Shapiro, Effective action of vacuum: semiclassical approach. Class. Quantum Gravity 25, 103001 (2008). arXiv:0801.0216 [grqc]

87. E.V. Gorbar, I.L. Shapiro, Renormalization group and decoupling in curved space. JHEP 02, 021 (2003). arXiv:hep-ph/0210388

88. E.V. Gorbar, I.L. Shapiro, Renormalization group and decoupling in curved space: II. The standard model and beyond. JHEP 06, 004 (2003). arXiv:hep-ph/0303124

89. S. Domazet, H. Stefancic, Renormalization group scale-setting in astrophysical systems. Phys. Lett. B 703, 1 (2011). arXiv: 1010.3585

90. N. Barnaby, N. Kamran, Dynamics with infinitely many derivatives: the initial value problem. JHEP 0802, 008 (2008). arXiv:0709.3968 [hep-th]

91. J.C. Fabris, A.M. Pelinson, F. de Oliveira Salles, I.L. Shapiro, Gravitational waves and stability of cosmological solutions in the theory with anomaly-induced corrections. JCAP 02, 019 (2012). arXiv:1112.5202 [gr-qc]

92. F. de O. Salles, I.L. Shapiro, Do we have unitary and (super)renormalizable quantum gravity below the Planck scale? Phys. Rev. D 89(8), 084054 (2014). Erratum: Phys. Rev. D 90(12), 129903 (2014). arXiv: 1401.4583 [hep-th]

93. A.M. Pelinson, F. de Oliveira Salles, I.L. Shapiro, Gravitational waves and perspectives for quantum gravity. Mod. Phys. Lett. A 29(30), 1430034-1 (Brief Review) (2014). arXiv:1410.2581 [grqc]

94. P. Peter, F. de Oliveira Salles, I.L. Shapiro, On the ghost-induced instability on de Sitter background. Phys. Rev. D 97(6), 064044 (2018). arXiv:1801.00063 [gr-qc]

95. J.D. Goncalves, T. de Paula Netto, I.L. Shapiro, Gauge and parametrization ambiguity in quantum gravity. Phys. Rev. D 97(2), 026015 (2018). arXiv:1712.03338 [hep-th]

96. B.L. Voronov, I.V. Tyutin, On renormalization of the einsteinian gravity (in Russian). Yad. Fiz. 33, 1710 (1981)

97. B. Slovick, Renormalization of Einstein gravity through a derivative-dependent field redefinition. Mod. Phys. Lett. A 33(02), 1850016 (2017). arXiv:1309.5945 [hep-th]

98. A.L. Maroto, I.L. Shapiro, On the inflationary solutions in higher derivative gravity with dilaton field. Phys. Lett. B 414, 34 (1997). arXiv:hep-th/9706179 